\title{
Adaptive immune responses to primary and secondary dengue virus infections
}

Ashley L. St. John $\mathbb{1}^{1,2,3 *}$ and Abhay P. S. Rathore ${ }^{3}$

Abstract | Dengue is the leading mosquito-borne viral illness infecting humans. Owing to the circulation of multiple serotypes, global expansion of the disease and recent gains in vaccination coverage, pre-existing immunity to dengue virus is abundant in the human population, and secondary dengue infections are common. Here, we contrast the mechanisms initiating and sustaining adaptive immune responses during primary infection with the immune pathways that are pre-existing and reactivated during secondary dengue. We also discuss new developments in our understanding of the contributions of $\mathrm{CD} 4^{+} T$ cells, $C D 8^{+} T$ cells and antibodies to immunity and memory recall. Memory recall may lead to protective or pathological outcomes, and understanding of these processes will be key to developing or refining dengue vaccines to be safe and effective.

Dengue is a viral illness that is spread primarily by Aedes mosquitoes and infects $\sim 390$ million people each year worldwide ${ }^{1}$. It is caused by dengue virus (DENV), which belongs to the family of Flaviviridae in the genus Flavivirus. Several other important human pathogens such as West Nile virus (WNV), Japanese encephalitis virus (JEV), yellow fever virus (YFV) and the recently re-emerged Zika virus (ZIKV) are closely related flaviviruses ${ }^{2}$. The DENV particle consists of a single-stranded positive-sense RNA enclosed within a capsid that is surrounded by an envelope containing the proteins $\mathrm{E}$ and $\mathrm{prM} / \mathrm{M}$. The three structural proteins (capsid, E and prM/M) plus seven non-structural proteins are transcribed and translated during viral replication $^{2}$, making them accessible to antigen-processing pathways within the cell, and the genome is synthesized through a double-stranded RNA intermediate. Humans can acquire symptomatic DENV infection more than once in a lifetime owing to the circulation of four antigenically distinct serotypes, DENV1-DENV4, which makes combating this disease complex. Immune memory responses are neutralizing and largely protective to viruses of the same serotype upon re-exposure. They are also cross-reactive; however, prior infection provides protection against all 4 serotypes for only 2-3 months, after which protection is serotype-specific ${ }^{3}$. When shortterm cross-protection wanes, patients with secondary DENV infections are at higher risk of severe disease ${ }^{4}$.

Clinically, dengue is defined as a febrile illness, but asymptomatic infections also occur and, on the basis of modelling of transmission dynamics, are estimated to constitute the majority of infections ${ }^{1}$ (FIG. 1a). After a mosquito bite, the incubation period is between 4 and 7 days before common flu-like symptoms arise, such as fever, nausea, muscle and joint pain and headache (FIG. 1 b,c). Mild cases, called dengue fever, are selfresolving. In some cases, as fever progresses, skin rashes and cutaneous bleeding manifestations may occur, such as petechiae and ecchymosis. Although DENV has been shown to be able to infect endothelial cells in vitro ${ }^{5}$, studies failed to identify wide-spread endothelial cell infection after fatal dengue ${ }^{6,7}$, and, furthermore, DENV was not detected in skin lesions distal to the site of virus infection in humans ${ }^{8}$. These findings indicate that vascular pathologies are likely to be primarily immunemediated. Nevertheless, virus-intrinsic virulence factors, which are not discussed in this Review, can participate in both pathogenesis and vascular leakage through multiple mechanisms and have been reviewed elsewhere".

A reduction in platelet counts (thrombocytopenia) also occurs during the acute phase of febrile illness. Most patients recover after defervescence. However, in a minority of patients, severe complications emerge around the time when fever subsides, and these can potentially be fatal. Severe disease, also known as dengue haemorrhagic fever (DHF) and dengue shock syndrome (DSS), is characterized by severe plasma leakage from the circulation and, occasionally, frank haemorrhage, organ failure and/or shock. Notably, immunological memory to a heterologous serotype alters the infection course such that viraemia likely peaks at higher levels, increasing the risk of developing DHF and/or 

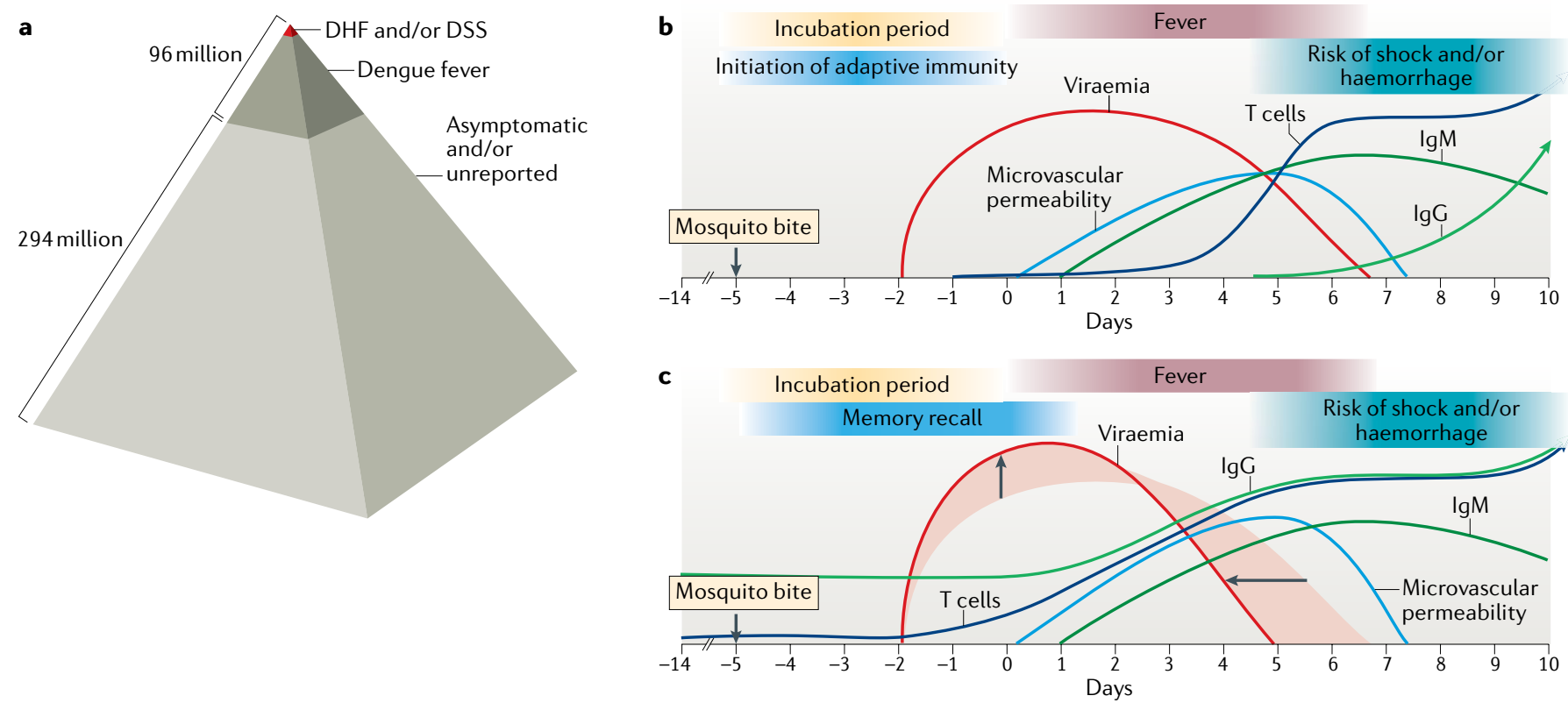

Fig. 1 | Time course of acute infection and immune responses during symptomatic dengue virus infection. a Asymptomatic cases account for the majority of yearly dengue virus (DENV) infections. Of symptomatic cases, only a small minority $(<5 \%)$ are severe, known as dengue haemorrhagic fever (DHF) and dengue shock syndrome (DSS) ${ }^{1}$. b | DENV infection begins with a mosquito bite, after which there is an incubation period of 4-6 days before fever develops. Peak viraemia correlates with high fever and coincides with the initiation of lgM and lgG production, which promote clearance of virus infection ${ }^{12,133}$. When viraemia is resolving or has subsided, some patients may experience haemorrhaging and/or shock that is thought to be immune-mediated ${ }^{134} \cdot \mathbf{c} \mid$ During secondary heterologous DENV infection, the period of viraemia is shorter, likely owing to cross-reactive immunity, including pre-existing antibodies. Cross-reactive memory $T$ cells promote an earlier and potentially stronger $T$ cell responses than that seen during primary infection ${ }^{59}$. Owing to the presence of pre-existing immune memory that may adversely affect immune responses to secondary heterologous DENV infection, the risk of severe dengue is higher in patients who experience secondary heterologous DENV infection ${ }^{4,85-87}$. Microvascular permeability, a clinical feature of dengue disease, usually becomes clinically apparent during the defervescent stage of disease ${ }^{135}$.

DENV serotypes

All four serotypes of dengue virus (DENV) share similar but distinct antigenic properties. Polyclonal sera raised to one DENV serotype can bind to all four DENV serotypes and may be minimally neutralizing but will efficiently neutralize and provide long-term protection against only the same serotype. DENV serotypes have $~ 50-60 \%$ sequence homology.
DSS $^{10,11}$, but viraemia has also been shown to subside more quickly ${ }^{12}$ (FIG. 1 C). However, adaptive immunity, involving multiple subclasses of antibodies and various subsets of B and T cells, can aid in the resolution of primary infection ${ }^{13,14}$ and prevent symptomatic infection by homologous DENV strains during secondary infection $^{3}$. The induction of such responses against all four DENV serotypes is the prime aim of vaccination. Here, we review our current knowledge of the adaptive immune response to DENV and the spectrum of protective to pathologic responses that can occur during a secondary infection as a result of pre-existing immune memory.

\section{Immunity to primary dengue infection}

Dengue immunity at the site of infection. At the site of the mosquito bite in the skin, various immune sentinels such as dendritic cells (DCs), Langerhans cells (LCs), macrophages and mast cells (MCs) are present. These sentinels are distributed throughout the skin at densities of $\sim 70-100 \mathrm{MCs}^{15}, \sim 60 \mathrm{CD} 11 \mathrm{c}^{+} \mathrm{DCs}$ and $\sim 80 \mathrm{CD} 63^{+} \mathrm{FXIIIA}^{+}$macrophages per $\mathrm{mm}^{2}$ of healthy human dermis ${ }^{16}$. Some skin cells, including DCs and macrophages, are known DENV infection target cell types, and either productive or abortive infection by DENV can trigger antiviral innate immune responses through the activation of pattern recognition receptors. For example, exposure of immature human myeloid
DCs to DENV induces activation and maturation of both infected DCs and uninfected bystanders, leading to the production of TNF and IFNa ${ }^{17,18}$, and abortive DENV replication in MCs triggers IFN $\alpha$ and chemokine responses $^{19}$. MCs are granulated immune cells that are preloaded with immune mediators and can respond to certain stimuli, including DENV, with a degranulation response within minutes ${ }^{19}$. This leads to the release of mediators such as TNF, which promotes to the retention of lymphocytes in draining lymph nodes (LNs), resulting in LN hypertrophy ${ }^{20}$. TNF also upregulates the expression of the adhesion molecule E-selectin on vascular endothelial cells. This, in turn, mediates the recruitment of monocytes into the skin, where they differentiate into $\mathrm{DCs}^{21}$. In mouse models, following subcutaneous DENV inoculation, DCs and LCs were found to migrate to the draining $\mathrm{LNs}^{22}$, which coincided with the early time points of $\mathrm{MC}$ activation ${ }^{19}$. As with other cutaneous infections and insults ${ }^{23}$, DC migration is understood to be an initiating event in adaptive immune responses to DENV (FIC. 2).

Initiation of dengue virus-specific adaptive immune responses. DENV infection starts in the human skin, yet the virus must achieve viraemia in order to infect new vector hosts for its spread. To accomplish this, DENV is lymphotropic (FIG. 2). Studies in primates and mice have 

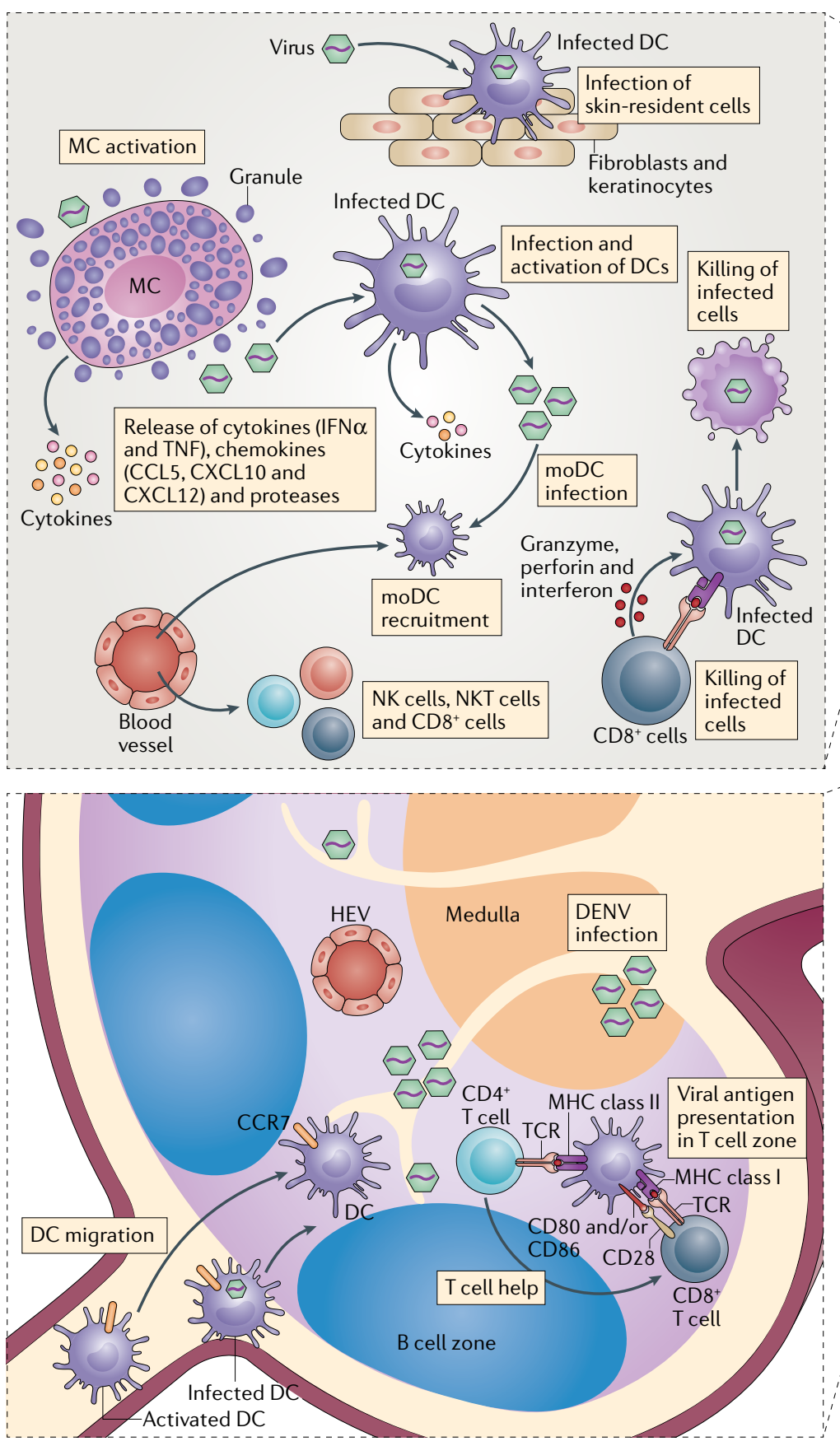

$(3)$

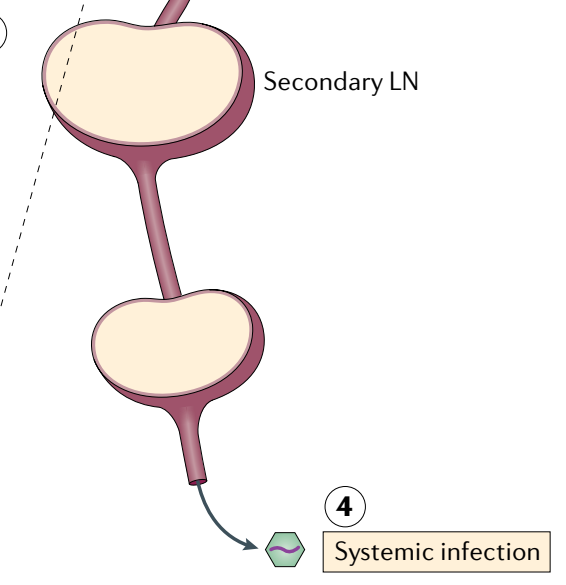

Fig. 2 | Initiation of anti-dengue virus immunity in the skin and draining lymph nodes. (1) Immune sentinel cells such as Langerhans cells (LCs), dendritic cells (DCs) and mast cells (MCs) are present in the epidermis and are the first immune cells to encounter dengue virus (DENV) after infection through a mosquito bite. MCs degranulate within minutes of DENV detection, releasing cytokines (for example, IFNa and TNF), chemokines (CCL5, CXCL10 and CXCL12) and proteases. The chemokines facilitate the recruitment of cytotoxic cells, such as $C D 8^{+} T$ cells (cytotoxic $T$ lymphocytes) (these have a Thelper $1\left(T_{H} 1\right)$ cell phenotype $)^{62}$, natural killer (NK) cells and natural killer $T$ (NKT) cells ${ }^{19}$, to the site of cutaneous infection. DCs, macrophages and/or monocytes, keratinocytes and possibly other cell types become infected, which leads to cytokine production by these infected cells in addition to the cytokines produced by MCs. Monocyte-derived DCs (moDCs), which are recruited to the site of infection, can serve as targets of infection and allow the amplification of the virus in the skin, while NK, NKT and CD8 ${ }^{+} T$ cells are able to kill DENV-infected cells and promote virus clearance. (2) Skin-resident dermal DCs and macrophages and recruited moDCs are targets of DENV infection and replication, and they also act as antigen-presenting cells. DCs migrate to the draining lymph node (LN) in a CCR7-dependent and CCL21-dependent manner. (3) In the LN, DENV-activated DCs upregulate expression of co-stimulatory molecules such as $\mathrm{CD} 80$ and $\mathrm{CD} 86$, and they present antigen to $\mathrm{CD} 4^{+}$and $\mathrm{CD} 8^{+} \mathrm{T}$ cells in $\mathrm{T}$ cell zones for the initiation of the adaptive immune response. Activated $C D 4^{+} T$ cells provide help to $C D 8^{+} T$ cells. Secondary $L N s$ also become infected sequentially following infection of the draining $\mathrm{LN}^{24}$ owing to the infection of cells such as DCs and macrophages ${ }^{30}$. LNs are amplification centres for DENV that the virus uses to progress to (4) systemic infection. HEV, high endothelial venule; TCR, T cell receptor. 
shown that DENV targets skin-draining LNs and infects cells that traffic through them, such as DCs and monocytes, to establish systemic infection ${ }^{24,25}$. In immunocompromised mice, the first cell types infected are $\mathrm{CD}_{103^{+}}$classical DCs (cDCs; non-lymphoid tissue type $^{26}$ ), LY6C ${ }^{-}$CD11 b $b^{+}$DCs (lymphoid tissue $\mathrm{cDCs}^{26}$ ) and macrophages, followed by monocyte-derived DCs $\left(\mathrm{LY}_{6 \mathrm{C}}{ }^{+} \mathrm{CD} 11 \mathrm{~b}^{+}\right)$, which are recruited to the infection site in substantial numbers ${ }^{27}$. Experimental inoculation of human skin explants with DENV revealed that the $\mathrm{CD}_{14}{ }^{+}$and $\mathrm{CD} 1 \mathrm{c}^{+}$subsets of dermal DCs, as well as LCs and dermal macrophages, were all infected ${ }^{28}$. In transwell migration assays, the subset of DENV-exposed DCs that migrated towards CCL19, an important chemokine for DC migration to LNs, was enriched in LCs and

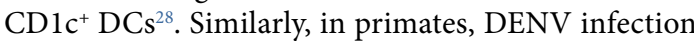
enhanced the numbers of $\mathrm{CD} 14^{+} \mathrm{CD} 16^{+}$monocytes in $\mathrm{LNs}^{29}$, and in immune-competent mice, $\mathrm{CD} 11 \mathrm{~b}^{+} \mathrm{CD} 11 \mathrm{c}^{+}$ DCs (lymphoid tissue cDCs) were found to be infected in $\mathrm{LNs}^{30}$, suggesting that DCs are the targets of DENV infection and contribute to its spread. However, further cell tracking studies are needed to establish definitively that tissue-resident infected DCs migrate from the skin to the LN. Yet, although infection proceeds through LNs, the functionality of these organs in generating specific immunity is apparently not substantially compromised because adaptive immunity to DENV is largely protective against viruses of the same serotype.

Despite the potential of DCs to become infected, they are also critical for antigen presentation to T cells in LNs, and there is evidence that the processing and presentation of DENV antigens influences downstream disease outcomes. For example, polymorphisms in transporter associated with antigen processing (TAP) and in certain HLA alleles have been associated with susceptibility or resistance to severe disease in humans ${ }^{31,32}$. DENVexposed human DCs upregulate activation markers, including CD80, CD86, HLA-DR and CD83, and the production of cytokines, including IFNa and $\mathrm{TNF}^{18}$. Infected or antigen-pulsed DCs can activate resting T cells from both naive and DENV-immune humans ${ }^{33,34}$, although data revealing the mechanisms of naive $\mathrm{T}$ cell activation during primary DENV infection are lacking. In vitro, DENV-exposed $\mathrm{CD} 14^{+} \mathrm{CD} 16^{+}$monocytes promote the differentiation of $\mathrm{B}$ cells into plasmablasts and enhance the secretion of IgG and $\operatorname{IgM}^{29}$. However, the contributions of specific subsets of DCs to either viral amplification or immune priming are currently unresolved. Despite evidence of protective immunity to homologous infections and the fact that DC activation pathways are transcriptionally enhanced during asymptomatic infections, DENV also antagonizes the antigen presentation pathways within DCs, including through its ability to antagonize type I and type II interferons ${ }^{35}$. Notably, type I and type II interferons also promote DC activation and antigen presentation to establish functional adaptive immune responses ${ }^{36,37}$.

A group of proteins, including IFN $\alpha$ and IFN $\beta$, that are

produced and secreted by cells activated by certain stimuli. including infection, for the purpose of host defence.

Often, these interferon proteins have antiviral function.

\section{Immune cells involved in dengue clearance}

Immune clearance of DENV in vivo is coordinated by multiple cell types and mechanisms. These include innate immune responses (such as production of type I and type II interferons) by the cells directly infected with DENV, cell killing by cytotoxic lymphocytes and production of neutralizing antibodies by B cells. In mice experimentally infected with DENV, MC activation in the skin was shown to be an initiating event in the recruitment of multiple subtypes of cytotoxic cells including natural killer (NK) cells, natural killer T (NKT) cells and CD8 ${ }^{+}$ $\mathrm{T}$ cells, which promoted the clearance of virus from the skin and limited the infection in draining $\mathrm{LNs}^{19}$. These cytotoxic lymphocytes are likely to be involved in the direct killing of DENV-infected target cells ${ }^{38}$, and they can produce cytokines such as IFN $\gamma$ that lead to a reduction in DENV production by infected cells ${ }^{39,40}$. Although an enhanced activation phenotype of both NK cells and $\mathrm{T}$ cells has been associated with severe disease in some studies ${ }^{41,42}$, it is not yet clear whether this observation represents a causal relationship with immune pathology ${ }^{43}$. However, the fact that DENV persists at the site of infection in the skin for $\sim 1$ week $^{28}$ suggests that the induction of adaptive immunity may be needed to fully resolve infection.

In humans, $\mathrm{T}$ cells are activated and polarized to a Thelper $1\left(\mathrm{~T}_{\mathrm{H}} 1\right)$ cell phenotype in their responses to $\mathrm{DENV}^{44}$, and serum cytokine responses after immunization with live-attenuated DENV vaccine candidate viruses were also found to be $\mathrm{T}_{\mathrm{H}} 1$ cell-polarized ${ }^{45}$. In terms of prevention of infection, it has been shown that neutralizing antibodies, the generation of which relies on the classical germinal centre reaction involving collaboration between $\mathrm{CD} 4^{+} \mathrm{T}$ helper cells and $\mathrm{B}$ cells, are able to block DENV infectivity, and their induction is the prime goal of DENV vaccination strategies ${ }^{46}$.

\section{New insights into protective $\mathrm{CD}^{+} \mathrm{T}$ cell responses} to dengue virus. The control of DENV infection in asymptomatic individuals correlates with increases in numbers of activated $\mathrm{CD} 4^{+} \mathrm{T}$ cells and is characterized by a transcriptional response of peripheral blood mononuclear cells that is indicative of antigen presentation and $\mathrm{T}$ cell activation ${ }^{47}$. Moreover, certain CD4-restricted HLAs, such as HLA-DRB1, have been linked to less severe DENV infection outcomes, suggesting that $\mathrm{CD} 4^{+} \mathrm{T}$ cells have a protective role ${ }^{48}$. However, in infection models using type I interferondeficient mice, $\mathrm{CD} 4^{+} \mathrm{T}$ cells were shown to be dispensable for virus clearance and the induction of antibody responses ${ }^{49}$, although it should be noted that type I interferons are required for DC activation to generate $\mathrm{CD}^{+} \mathrm{T}$ cell immunity ${ }^{50}$ and that they promote the cross-priming of $\mathrm{CD}^{+} \mathrm{T}$ cells by $\mathrm{DCs}^{51}$. IFN $\gamma$, which is crucial for the cytotoxic function of $\mathrm{CD}^{+} \mathrm{T}$ cells, is also produced in large quantities by activated $\mathrm{CD}^{+}$ $\mathrm{T}$ cells and is a major contributor to $\mathrm{T}$ cell help ${ }^{37}$. It is possible that $\mathrm{CD} 4^{+} \mathrm{T}$ cells are more functionally important in interferon-sufficient systems or in humans, where the virus is more effectively contained than in immunocompromised mice.

$\mathrm{CD}^{+}{ }^{+} \mathrm{CD} 25^{\text {hi }} \mathrm{FOXP}^{+}$regulatory $\mathrm{T}\left(\mathrm{T}_{\text {reg }}\right)$ cells also expand during acute DENV infection, but their functional role in this context is unclear. Although increased $\mathrm{T}_{\text {reg }}$ cell frequencies and increased $\mathrm{T}_{\text {reg }}$ cell to effector $\mathrm{T}$ cell ratios were associated with milder disease in 
Heterologous DENV

infection

A dengue infection with one dengue virus (DENV) serotype following a primary infection by a different DENV serotype

For instance, DENV1 infection followed by DENV2 infection. one study ${ }^{52}$, no association was found with viraemia or disease severity in a different cohort ${ }^{53}$.

Recently, $\mathrm{T}$ follicular helper $\left(\mathrm{T}_{\mathrm{FH}}\right)$ cells were found to be expanded during acute DENV infection, particularly in humans with secondary heterologous DENV infection and in mice with secondary homologous DENV infection ${ }^{34,54}$. The functional significance of $T_{F H}$ cells during DENV infection has not been shown in humans, but in general $\mathrm{T}_{\mathrm{FH}}$ cells are able to traffic between germinal centres, promoting the diversification of $\mathrm{T}$ cell help $\mathrm{p}^{55}$. In the context

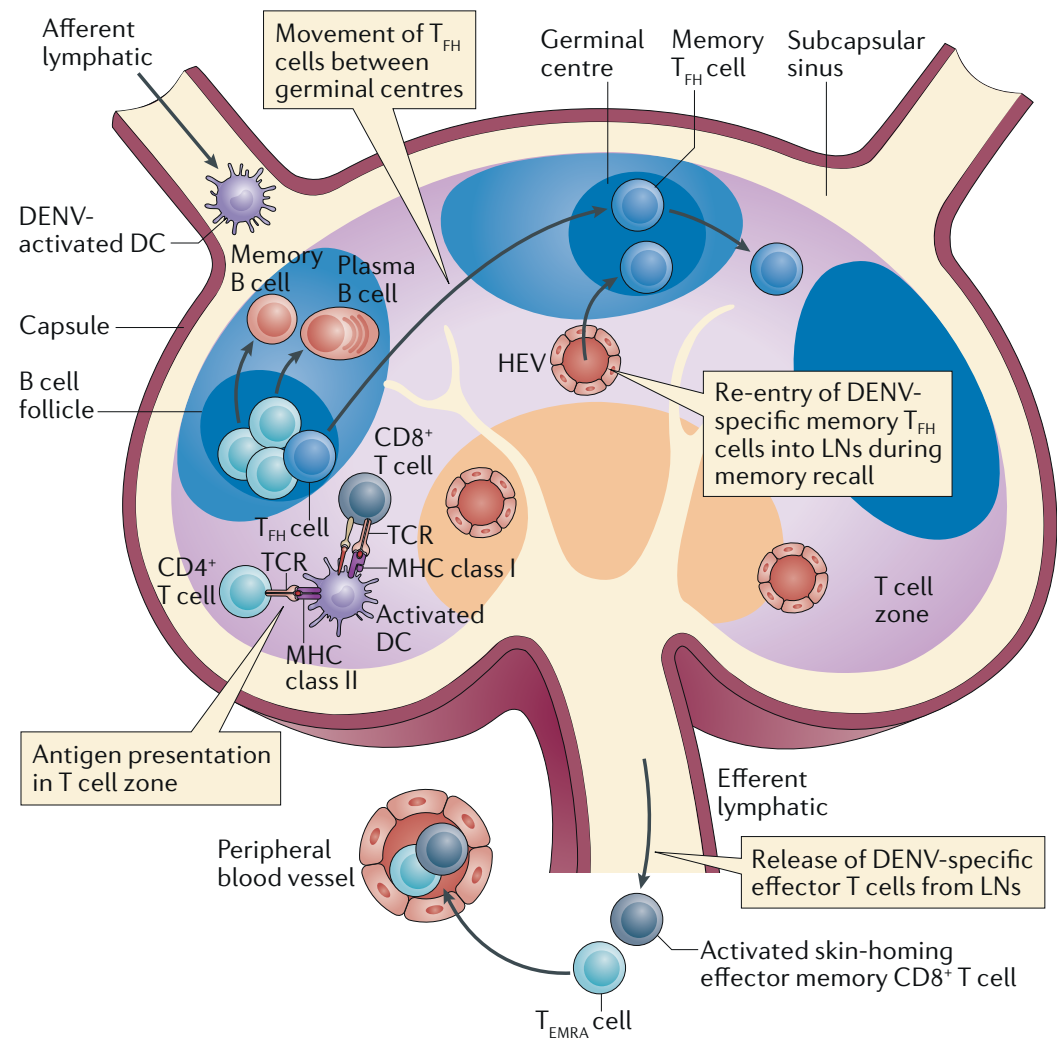

Fig. 3 | Adaptive T cell responses during dengue virus infection. Activated dendritic cells (DCs), some having been infected by dengue virus (DENV) in the skin, arrive in the draining lymph node (LN) through afferent lymphatics, where they present antigen to both $\mathrm{CD}^{+}$and $\mathrm{CD} 8^{+} \mathrm{T}$ cells for the initiation of the adaptive immune response. CD4 ${ }^{+}$ $T$ cells, activated by DENV antigen presented by DCs, have the potential to become $T$ follicular helper $\left(T_{F H}\right)$ cells. These migrate from $T$ cell zones to the periphery of the B cell follicle in the $\mathrm{LN}$, where they subsequently participate in the germinal centre reaction and promote the development of DENV-specific memory B cells and plasma cells. DENV-specific memory T cells express surface markers such as CD44 (in mice) and CD45RO (in humans) ${ }^{34} . T_{F H}$ cells are able to traffic to new follicles and to re-enter germinal centres ${ }^{34}$. During a secondary DENV infection, memory $C D 8^{+} T$ cells acquire a skin-homing phenotype $\left(\mathrm{CXCR} 3^{+} \mathrm{CCR}{ }^{+} \mathrm{CLA}^{+}\right)^{62}$. These cells can exit the $\mathrm{LN}$ through efferent lymphatics and re-enter circulation and theoretically have the potential to home to the skin for clearance of DENV. Similarly, $C D 4^{+} T$ cells can also acquire a cytotoxic phenotype (CD45RA ${ }^{\text {hi }} \mathrm{CCR} 7^{\text {low }} \mathrm{GPR} 56^{+} \mathrm{CX}_{3} \mathrm{CR} 1^{+}$granzyme ${ }^{+}$) and are known as T effector memory RA $\left(T_{\text {EMRA }}\right)$ cells, and these cells are also thought to contribute to DENV clearance ${ }^{57,58}$. During secondary DENV infection, antigen-specific plasma $B$ cells undergo clonal expansion in the LNs. Memory $\mathrm{T}_{\mathrm{FH}}$ cells, which circulate in the blood and are able to re-enter LN germinal centres, can facilitate the clonal expansion of B cells and thereby enhance antibody production and affinity maturation during a secondary DENV infection ${ }^{34}$. The recent observations regarding $T$ cell phenotypes, functions and trafficking during DENV infection suggest that $T$ cells can play a substantial role in providing protection against DENV. GPR56, G protein-coupled surface receptor 56; HEV, high endothelial venule; TCR, T cell receptor. of DENV infection, $\mathrm{T}_{\mathrm{FH}}$ cells promote affinity maturation of DENV-specific antibodies, leading to higher avidity antibodies with greater neutralizing capacity in mice during homologous and flavivirus-cross-reactive challenges $^{34}$ (FIG. 3). In humans, effector $\mathrm{CD} 4^{+} \mathrm{T}$ cells activated by DENV infection can also develop cytotoxic function ${ }^{40,56}$. These are likely $T$ effector memory RA cells $\left(\mathrm{T}_{\mathrm{EMRA}}\right.$ cells), which have an effector memory phenotype (CD45RA $\left.{ }^{\text {hi }} C C R 7^{\text {low }}\right)$. DENV-specific $C D 4^{+} \mathrm{T}_{\mathrm{FMRA}}$ cells were shown to express the adhesion protein $G$ proteincoupled surface receptor 56 (GPR56), the chemokine receptor $\mathrm{CX}_{3} \mathrm{CR} 1$ and the serine protease granzyme and to be a substantial source of IFN $\gamma^{57}$. Although cells of this phenotype were restricted to certain HLAs, these cells may have been clonally expanded, and their cytotoxic function may be protective ${ }^{58}$ (FIG. 3).

$C D 8^{+} T$ cell responses during primary dengue infection. For patients with acute primary DENV infection, $\mathrm{CD} 8^{+} \mathrm{T}$ cell responses peak around the day of defervescence or slightly before (FIG. 1 b), as shown by DENV-specific tetramer staining ${ }^{59}$. Immune memory also persists following contraction of the $\mathrm{T}$ cell response ${ }^{59}$. During the febrile stage of illness, a massive expansion of activated $\mathrm{CD} 8^{+} \mathrm{T}\left(\mathrm{CD} 38^{+} \mathrm{HLA}-\mathrm{DR}^{+}\right)$cells is observed. However, when analysed ex vivo, these cells were found to be exhausted, unresponsive to stimulation with pooled DENV peptides and with limited ability to produce IFN $\gamma$ and IL-2 in response to stimulation. Exhaustion was attributed to T cell receptor (TCR) insufficiency because the cells were still responsive to TCR-independent stimuli, such as ionomycin but not to polyclonal TCR-dependent stimulation (beads coated with anti-CD3 and anti-CD28 antibodies) ${ }^{60}$. It is also noteworthy that high levels of both IFNa and IFN $\gamma$ were found in the plasma of patients during early primary DENV infection, suggesting that cells other than $\mathrm{T}$ cells were the major source of IFN $\gamma^{61}$. Phenotypically, $\mathrm{CD}^{+} \mathrm{T}$ cells of DENV-infected humans have a skinhoming phenotype (FIG. 3) typified by the expression of the chemokine receptors CXCR3 and CCR5 and cutaneous lymphocyte-associated antigen (CLA). This was potentially imprinted by the process of antigen presentation from skin-derived $\mathrm{DCs}^{62}$. A subset of $\mathrm{CLA}^{+} \mathrm{CD}^{+}$ $\mathrm{T}$ cells also expressed the chemokine receptor CXCR6, which may promote homing to the liver ${ }^{62}$. However, it is not yet clear whether the abundance of these cells in patients with symptomatic dengue is indicative of effective virus clearance.

Epitopes for $C D 4^{+}$and $C D 8^{+} T$ cell recognition. Views on the functional influence of $\mathrm{T}$ cell responses in DENV infection may lack consensus, but efforts to identify $\mathrm{T}$ cell epitopes are likely to clarify which virus-directed immune responses are protective and whether any are also harmful. $\mathrm{T}$ cell responses, and in particular $\mathrm{CD} 8^{+}$ $\mathrm{T}$ cell responses, are generally assumed to be directed primarily at antigenic epitopes of non-structural proteins, as these are abundant in the host cytosol during virus replication. Regions of the genome that are predicted to be human $\mathrm{CD} 8^{+} \mathrm{T}$ cell epitopes appear to be lost at higher rates in the DENV genome than in closely 
Homologous DENV

infection

This refers to a secondary dengue virus (DENV) infection with the same serotype that caused the primary infection. For example, DENV1 infection followed by a secondary DENV 1 infection.

T effector memory RA cells ( $T_{\text {EMRA }}$ cells). Terminally differentiated antigen-specific memory $T$ cells that re-express CD45RA. These cells have been identified in both $\mathrm{CD}^{+}$ and $C D 8^{+} T$ cell compartments and can have a cytotoxic phenotype.

Original antigenic sin Reactivation and expansion of an immune memory response that was formed in response to a previous infection upon exposure to a second infection from a pathogen that has similar but distinct antigenic properties to that of the first pathogen. This results in a skewed and potentially suboptimal immune response generated during infection by the second pathogen. related viruses (such as WNV) that have a broader host species range and historically lower circulation in human populations ${ }^{63}$, indirectly suggesting a population-level selection against DENV strains that induce cytotoxic T lymphocyte responses.

While some groups have shown that responses against DENV non-structural protein 3 (NS3) are immunodominant for both $\mathrm{CD}^{+}$and $\mathrm{CD} 4^{+} \mathrm{T}$ cells ${ }^{64}$, another study that aimed to identify $\mathrm{T}$ cell epitopes agreed that responses to NS3 epitopes are dominant for $\mathrm{CD}^{+} \mathrm{T}$ cells but identified few NS3 epitopes for $\mathrm{CD} 4^{+} \mathrm{T}$ cells. By contrast, they observed that epitopes recognized by $\mathrm{CD} 4^{+} \mathrm{T}$ cells were primarily located in the same structural and secreted proteins that are also targets for antibodies, namely, the E, capsid and NS1 proteins ${ }^{65}$. Recently, a study examined $\mathrm{T}$ cell epitopes identified in patient cohorts from multiple endemic areas that respond to highly prevalent human MHCs (for example, HLA-DRB1). There, DENV-specific $\mathrm{CD}^{+} \mathrm{T}$ cells predominantly recognized epitopes of the viral capsid as well as NS3 and NS5 proteins ${ }^{66}$. Together, these studies highlight $\mathrm{T}$ cell epitopes that may be widely recognized in the human population and indicate that both non-structural and structural proteins can contain immunodominant $\mathrm{CD}^{+}$and $\mathrm{CD} 8^{+} \mathrm{T}$ cell epitopes.

$B$ cells and antibody responses after primary infection. Within days following infection, plasmablasts and titres of DENV-specific antibodies increase in the blood ${ }^{67}$. However, comparisons between patients with asymptomatic or clinically apparent DENV infection showed that plasmablast pathways are less activated in individuals with asymptomatic DENV infection, challenging the assumption that plasmablast induction is necessarily associated with DENV control ${ }^{47}$. Nevertheless, neutralizing antibody responses are induced by DENV infection and contribute to its clearance. Using human serum depleted of functional IgM, it was shown that at 4-7 days after the onset of fever, the IgG response already accounted for $\sim 50 \%$ of the neutralizing capacity of DENV-specific immune serum. Unlike other viral infections such as HIV infection, which can induce hypergammaglobulinemia ${ }^{68}$, only a slight increase in polyclonal B cell activation was observed ${ }^{67}$. In general, DENV-specific B cells were highly serotype-specific in primary infection ${ }^{69}$.

It is thought that $\sim 30$ antibodies are needed to bind to the flavivirus virion for neutralization, a number that is lower in the presence of complement ${ }^{70}$. In addition to the targeted epitope, a key property of neutralizing antibodies is a high affinity for their cognate antigen, which allows neutralization ${ }^{71}$. Although most DENVspecific epitopes that have been identified are linear (likely owing to technical reasons), a number of recent studies have shown that antibodies against the quaternary virus structure that spans the E dimer are highly neutralizing against both homotypic and heterotypic DENV serotypes ${ }^{72,73}$. Another domain of interest for virus neutralization is the EDIII region of the E protein because it has been shown that antibodies that bind to certain epitopes of EDIII can neutralize multiple DENV serotypes ${ }^{74}$. Under experimental conditions, the temperature, $\mathrm{pH}$ and overall dynamic movement of the viral particle expose hidden neutralizing epitopes that are not easily accessed by antibodies when the virus is in its mature compact conformation ${ }^{75}$. Alternate E protein structures that occur during virus fusion with the endosomal membrane, which have not yet been solved, may provide additional epitopes for virus neutralization.

\section{Contrasting primary and secondary infection}

Secondary homologous dengue infection. Decades ago, in seminal human challenge studies, it was demonstrated that reinfection of humans with a homologous DENV strain provided total protection from symptomatic dengue, even after 18 months, which was the longest interval tested ${ }^{3}$. However, in a recent large cohort study ( $>2,800$ patients), it was found that immunity to homologous serotypes may not always be fully sterilizing, as 4 patients had probable homologous reinfections ${ }^{76}$. However, further studies are needed to identify the causes for weak protection in this particular study, including the possibility of underlying diseases or coinfections. The previous lack of evidence for homologous reinfection supports the view that natural immunity to DENV is mostly protective in healthy humans, even if not fully sterilizing. Numerous studies also demonstrate effective reactivation of memory $\mathrm{T}$ cells by peptides from homologous DENV serotypes ${ }^{48,56,77,78}$, which presumably contribute to protection and to the boosting of antibody responses. In mice, antibody quality improves (in terms of avidity and neutralizing capacity) upon reinfection with a homologous DENV serotype ${ }^{34}$.

Memory recall during heterologous dengue infections. During secondary DENV infection, the magnitude and kinetics of $\mathrm{B}$ and $\mathrm{T}$ cell activation responses are enhanced even though memory recall involves the activation of serotype cross-reactive memory cells. In experimental mouse models, serotype-specific T cell responses towards the second infecting strain arise following secondary infection, suggesting that both naive $\mathrm{T}$ cells and serotype-cross-reactive memory $\mathrm{T}$ cells are activated $^{79}$. A significant expansion of plasmablasts is observed ( 1,000-fold over baseline in healthy adults), accounting for up to $30 \%$ of the total circulating lymphocyte population. This is a unique response to DENV; in comparison, repeated booster doses of influenza vaccines evoke a more measured plasmablast response ( $6 \%$ of total circulating B cells $)^{80}$. These memory B cells are DENV-specific and rapidly secrete large amounts of virus-specific IgG ${ }^{81}$. Antibodies and memory B cells that are detected during the acute phase of secondary DENV infection also show cross reactivity for multiple serotypes $^{82,83}$, as do T cell responses ${ }^{42}$, which could lead to original antigenic sin. Unlike plasmablasts, the frequencies of DENV-specific CD8 ${ }^{+} \mathrm{T}$ cells in the blood appear similar during primary and secondary DENV infection ${ }^{59,84}$; however, this interpretation is based on comparisons for T cells targeting only a few specific peptide-HLA complexes. A key distinction between primary and secondary infection is the presence of pre-existing antibodies, which are thought to increase infection and disease severity, and cross-reactive $\mathrm{T}$ cell populations that can 


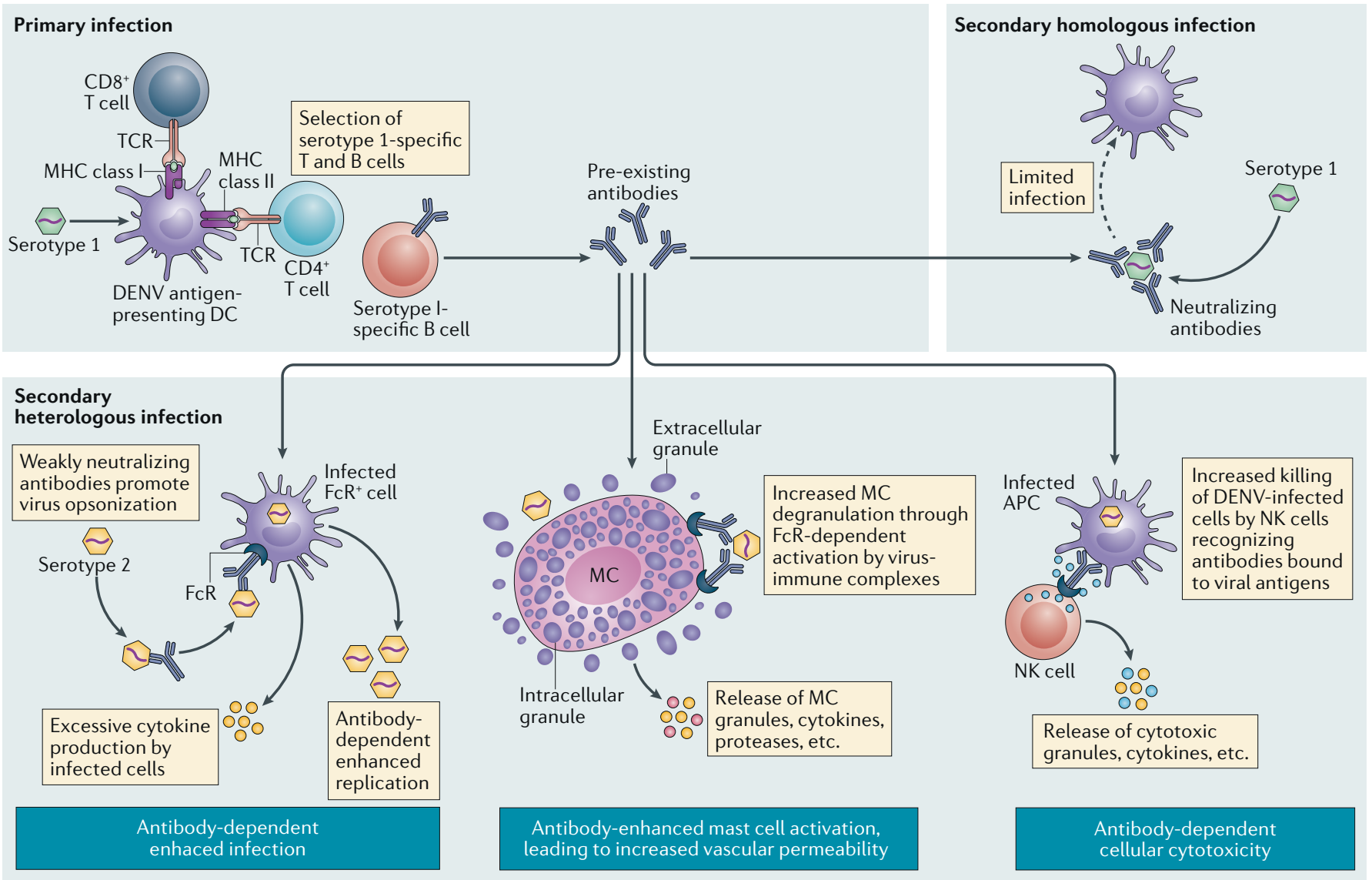

Fig. 4 | Antibody-dependent pathologies during dengue virus infections. Pre-existing antibodies against dengue virus (DENV) interact with many different cell types, and there are several theories for how these can contribute to severe disease. After primary infection, serotype-specific high-affinity antibodies are produced that, when present at optimal concentrations, can neutralize DENV. During a secondary homologous infection, the titres of pre-existing antibodies may be boosted and lead to effective neutralization of DENV so that infection is limited (indicated by dashed arrow). However, during secondary heterologous infection, pre-existing cross-reactive, sub-neutralizing antibodies may lead to opsonization of virus particles and enhanced uptake by various immune cells such as monocytes and macrophages via crystallizable fragment $-\gamma$ receptors (Fc $\gamma$ Rs), resulting in increased virus replication, a phenomenon termed antibody-dependent enhancement (ADE). In infected immune cells, the increased viral burden owing to ADE may produce excessive cytokines, leading to a cytokine storm ${ }^{42,136}$. Similarly, mast cells (MCs) can also recognize antibody-bound virus particles in a crystallizable fragment receptor $\left(F_{c} R\right)$-dependent manner, resulting in increased degranulation of pre-stored granule-associated cytokines and proteases and de novo synthesized soluble mediators such as cytokines. These cytokines and proteases can also lead to increased vascular permeability and leakage during DENV infection. Antibodies may also contribute to pathology during secondary DENV infection through antibody-dependent cellular cytotoxicity, where antibodies bind to the surface of DENV-infected cells, resulting in direct lysis of infected cells by cytotoxic natural killer (NK) cells ${ }^{112}$. The release of cytotoxic granules and cytokines by NK cells may also contribute to a cytokine storm, and the tissue damage associated with infection clearance may exacerbate the pro-inflammatory environment ${ }^{112}$. APC, antigen-presenting cell; DC, dendritic cell; TCR, T cell receptor.

provide cross-protection but also potentially worsen disease outcomes owing to original antigenic sin or other mechanisms, discussed below.

Antibody-mediated dengue pathologies. Approximately 40 years of research have amassed a wealth of literature supporting the hypothesis that antibodies increase dengue severity and augment virus replication, a phenomenon termed antibody-dependent enhancement (ADE). This was observed in cohorts of patients in Thailand ${ }^{4}, \mathrm{Cuba}^{85}$, Vietnam ${ }^{86}$ and Nicaragua ${ }^{87}$ where pre-existing immunity was determined by serological studies. The observation that patients with prior DENV immunity were more likely to develop DHF and/or DSS has been attributed to ADE, which involves the opsonization of DENV particles that are bound in immune complexes with non-neutralizing antibodies (FIG. 4). Crystallizable fragment $(\mathrm{Fc})$ receptor $(\mathrm{FcR})$-bearing cells that are targets for DENV infection, such as DCs and monocytes, can experience ADE, leading to high levels of viral replication. This phenomenon has been shown in vitro and in vivo in immunocompromised mice and in primates ${ }^{88-91}$. Antibodies also appear to increase infection rates of immune cell types that are usually not efficiently infected, such as $\mathrm{MCs}^{92}$. Antibodies against certain, but not all, epitopes, including the 
DENV E protein fusion loop, often have infectionexacerbating properties ${ }^{93}$. Similarly, several ADEinducing antibodies have been identified that target epitopes on the prM portion of the DENV M protein, which is cleaved during virus maturation, so these antibodies can make immature DENV particles infectious ${ }^{89,94}$.

As antibody titres and their affinity (or avidity in the context of polyclonal sera) vary, the neutralizing versus enhancing properties of these antibodies also fluctuate. In vitro, even neutralizing antibodies can have enhancing properties when present at low concentrations, and weakly neutralizing cross-reactive antibodies can also neutralize when present at high concentrations ${ }^{95}$. Consistent with this, in a Nicaraguan patient cohort, it was shown that the risk of severe dengue is elevated only when pre-existing antibody titres are within a narrow intermediate range, while individuals with either higher or lower titres showed no increased risk of DHF and/or $\mathrm{DSS}^{87}$. Beyond the potential of increasing virus uptake and subsequent replication, viral replication activates intracellular antiviral innate immune pathways, which may contribute to the production or release of potentially harmful inflammatory products. The intracellular signalling cascades induced by the binding of immune complexes to FcRs can also lead to immune activation and cytokine production or, alternatively, increase the production of virus owing to their effect on signalling pathways involved in virus replication or host defence ${ }^{96,97}$. It has been suggested that increased plasmablast frequencies during heterologous DENV infection lead to worse clinical outcomes, possibly owing to ADE of DENV, promoted by weakly neutralizing cross-reactive antibodies $^{82,98}$. A similar phenomenon in which an expansion of plasmablasts occurs is observed in patients with systemic lupus erythematosus and is driven by increased levels of IL-10 secreted by activated monocytes, as IL-10 promotes plasmablast differentiation ${ }^{29,99}$. This mechanism possibly underlies plasmablast expansion during severe dengue, as blockade of IL-10 derived from DENV-exposed monocytes in vitro limits plasmablast proliferation ${ }^{29}$. Although it is uncertain how plasmablast expansion is linked to severe disease, it may be due to an enhanced production of low-affinity cross-reactive antibodies.

Particular isotypes of antibodies may also influence disease severity. For example, it has been shown that a high IgG1:IgG2 ratio and the presence of afucosylated IgG1, which binds to the activating FcR Fc $\gamma$ RIIIA, are risk factors for thrombocytopenia (a reduction in platelets) in individuals with DENV infection ${ }^{100}$. The absence of the fucose sugar group increases the affinity of antibodies for the FcR and may therefore lead to an enhancement of the interactions between DENV immune complexes and $\mathrm{Fc}_{\mathrm{R}} \mathrm{RIIIA}^{+}$immune cells, such as NK cells, monocytes, macrophages and DCs. However, surprisingly, injection of afucosylated antibodies alone, without DENV, in human FcR knock-in mice was sufficient to deplete platelets, suggesting that DENV is not required for this mechanism of platelet reduction, which was also dependent on Fc $\gamma$ RIIIA and Fc $\gamma$ RIIA $^{100}$. Interestingly, a high IgG1:IgG2 ratio also indicates a $\mathrm{T}_{\mathrm{H}} 2$ cell-polarized immune profile, whereas DENV clearance is thought to require $\mathrm{T}_{\mathrm{H}} 1$ cell immunity.
One specific scenario in which the increased risk of severe dengue infection owing to pre-existing immunity is particularly striking is in the context of maternal immunity. Maternal antibodies, which are actively transported across the placenta or provided in breast milk and taken up by the gut through neonatal FcR-dependent mechanisms ${ }^{101}$, can exacerbate disease severity in infants. In a prospective study of infants of mothers immune to DENV, susceptibility to DENV increases as the blood concentrations of maternal antibodies wane, suggesting that high titres of anti-DENV antibodies immediately following birth provide some protection ${ }^{102}$. However, at 4-12 months of age, low concentrations of anti-DENV antibodies can potentially contribute to $\mathrm{ADE}^{102}$. The sequence of maternal and infant infections by different DENV serotypes is also believed to influence ADE in infants. For example, maternal antibodies against the DENV2 and DENV4 serotypes were associated with severe disease in newly infected infants in one epidemiological study ${ }^{103}$, while maternal antibodies against the DENV3 serotype were not ${ }^{104}$. This suggests that antigen-specific properties of maternal antibodies play a role in disease exacerbation in infants. In DENV infection models in immunocompromised mice, heterologous maternal immunity also led to exacerbated disease in the progeny that were infected several weeks after birth ${ }^{105}$. Most of that effect was subsequently shown to be dependent on antibodies that were transferred in breast milk ${ }^{106}$. However, studies are needed to ascertain the concentrations of DENV-specific antibodies in human breast milk.

Additional evidence for ADE in DENV infection has come from an experimental system using immunocompromised mice ${ }^{90,107}$. In this system, animals were injected with DENV cross-reactive antibodies before infection, which resulted in ADE in vivo and increased disease severity. In humans, it was recently shown that immunity to an inactivated JEV vaccine led to augmented titres of the live-attenuated YFV vaccine during a secondary vaccination. Although the antigenic similarities of viruses from differing serocomplexes differ substantially from those between the more closely related DENV serotypes, this study shows that $\mathrm{ADE}$ in response to infection with flaviviruses can occur in humans ${ }^{108}$. Recent evidence, based on retrospective confirmation of DENV serological status also suggests that the first licensed dengue vaccine, Dengvaxia (Sanofi Pasteur), is associated with an increased risk of severe disease and hospitalization owing to DENV infection in individuals who had not encountered DENV previously ${ }^{109}$. The leading theory, although yet unproven, is that the vaccine can sometimes induce an ADE-type response when natural infection occurs ${ }^{110}$.

One caveat in assuming that ADE is the primary culprit causing increased disease severity is that increased virus titres to secondary heterologous infections are not always observed in humans, unlike in mouse infection models. Indeed, it has been shown that viraemia is cleared faster in patients with secondary heterologous DENV infection than in those with primary infection $^{12}$, but it cannot be known for certain whether viral titres might peak at higher levels before monitoring the patients or in an inaccessible physiological compartment 
Reverse Arthus reaction

The immune complex-mediated vasculitis in a type III

hypersensitivity reaction that results from the injection of antibodies into the skin

following passive infusion of antigen.

Antibody-dependent cellular cytotoxicity An immune phenomenon where crystallizable fragment (Fc) receptor-bearing cytotoxic immune cells can recognize and lyse antibody-coated, antigen-expressing cells Antigens that can lead to antibody-dependent cellular cytotoxicity may arise owing to infection such as lymphoid tissues where virus could possibly persist even after viraemia has resolved. Yet, there are also mechanisms of antibody-mediated pathology during DENV infection that are independent of ADE. Antibodies also interact with other cell types beyond DCs, including MCs and NK cells, in ways that do not require these target cells to become infected themselves. MCs, for example, can interact with both IgG and IgE owing to the expression of various subclasses of Fc $\gamma$ Rs and the FceR. Elevated DENV-specific IgE titres have also been associated with severe disease ${ }^{111}$. Antibody-mediated enhanced MC degranulation has been observed in mice with a secondary heterologous DENV infection. This was dependent on the binding of DENV-specific IgG to Fc $\gamma$ RIII, the activating FcR that is expressed on mouse MCs (human MCs express multiple homologous activating FcRs) ${ }^{92}$. This study indicates that MC degranulation, mediated by immune complexes consisting of IgG and DENV virions, occurs, which is similar to the reverse Arthus reaction ${ }^{92}$ (FIG. 4). Consistent with this, patients with DHF with secondary infection have been found to have enhanced serum levels of the enzyme chymase, which is a biomarker for MC activation, compared with patients with primary infection, supporting the theory that antibody-enhanced MC activation occurs in humans with secondary severe disease owing to secondary heterologous DENV infection ${ }^{30}$. Antibodies may also directly contribute to cell lysis via antibody-dependent cellular cytotoxicity ${ }^{112}$ (FIG. 4). As with antibody-enhanced MC responses ${ }^{92}$, this NK cellmediated activity has the potential to aid virus clearance but can also increase tissue damage.

$T$ cell responses during secondary infection. There is an increasing number of reports that suggest an important role for T cells in immune-recall responses to secondary DENV infection. Similar to antibody responses, there is evidence that $\mathrm{T}$ cell responses can aid viral clearance ${ }^{34,57,64}$ but potentially also exacerbate immune pathology ${ }^{42,113}$ (FIG. 5).

Evidence for heterologous memory $\mathrm{CD}^{+} \mathrm{T}$ cell activation during secondary DENV infection is provided by the fact that $\mathrm{T}$ cells from DENV-immunized donors, when stimulated with heterologous DENV antigens, respond with cytokine production ${ }^{78}$. Moreover, exposure of T cells from DENV-immune donors to allogenic DCs pulsed with DENV antigen leads to an upregulation of the $\mathrm{T}$ cell activation marker HLA-DR on memory T cells, indicating memory recall ${ }^{34}$. Furthermore, in a study of patients who were DENV1 and DENV3 immune, it was shown that their DENV-specific $\mathrm{CD}^{+} \mathrm{T}$ cell repertoire following primary infections was highly cross-reactive ${ }^{77}$. In immunocompromised mice, $\mathrm{CD}^{+} \mathrm{T}$ cells promoted protection from heterologous and homologous DENV reinfection ${ }^{114,115}$. However, initial studies that examined DENV-specific $\mathrm{CD}^{+} \mathrm{T}$ cell responses in hospitalized patients with secondary heterologous DENV infection showed that weak-affinity $\mathrm{CD} 8^{+} \mathrm{T}$ cell responses were associated with DHF and/or DSS. This phenotype was postulated to be indicative of original antigenic sin, where cross-reactive heterologous immunity may lead to an expansion of weak-affinity $\mathrm{T}$ cells that are inefficient in clearing DENV infection, while producing excessive cytokines, leading to immune pathology ${ }^{43}$ (FIG. 5). During heterologous reinfection, differences in the presented peptide sequences may also elicit a unique activation response by the TCR of memory $\mathrm{T}$ cells, altering the phenotype or function of these cells ${ }^{116}$. Consistent with this, when $\mathrm{T}$ cells from donors who had a primary DENV infection were stimulated with either homologous or heterologous peptides, differing T cell activation phenotypes in terms of cytokine production and TCR signalling were observed ${ }^{77,117}$. However, it should be noted that although these studies support the hypothesis that many $\mathrm{T}$ cell epitopes are conserved for DENV strains, other studies did not identify an altered $\mathrm{T}$ cell phenotype that was associated with the reactivation of T cells to heterologous DENV antigens or with severe disease $^{64}$ or correlations between $\mathrm{T}$ cell activation and the onset of vascular leakage ${ }^{118}$. These studies question the relevance of memory $\mathrm{T}$ cells to DENV pathology during secondary infections.

However, a recent study in humans showed a bias of T cell responses towards certain TCR-MHC-peptide interactions during DENV infection, which may explain the expansion of low-affinity T cells and T cell-mediated immune pathology. Specifically, it was shown that a certain TCR $\beta$ (TR $\beta$ V11-2 $2^{+}$) complexed with a DENV2 NS3derived peptide-loaded MHC (HLA-A11:01) resulted in the expansion of $\mathrm{CD}^{+} \mathrm{T}$ cells with weak affinity during sequential infections with heterologous serotypes. During heterologous DENV infection, these T cells may compete with protective $\mathrm{T}$ cells that have stronger affinity, resulting in inefficient control of the virus and in immune pathology $y^{113}$. Furthermore, as the propensity to respond to this peptide is encoded in the germ line, varied immune responses to DENV may be due to differences in genetic backgrounds in humans. Overall, it remains unclear whether cross-reactive $\mathrm{CD}^{+} \mathrm{T}$ cells have a protective function during secondary heterologous infection or whether they contribute to pathology. Because $\mathrm{CD}^{+} \mathrm{T}$ cells are also critical for the development of $\mathrm{CD}^{+}$memory $\mathrm{T}$ cells ${ }^{119}$, it will be important to understand the role of DENV-specific CD4 ${ }^{+}$ $\mathrm{T}$ cells in shaping $\mathrm{CD}^{+} \mathrm{T}$ cell responses during DENV infection.

\section{Pre-existing flavivirus immunity}

Phylogenetic analysis shows that flaviviruses are closely related and have a similar genomic organization ${ }^{120}$. Their proteins are also antigenically similar, as shown using neutralization and antibody binding tests of polyclonal antisera $^{121}$. Therefore, in addition to serotype crossreactive responses, there is the potential of serocomplexcross-reactive immune responses when pre-existing immunity is present to flaviviruses such as WNV, JEV, YFV, ZIKV and others ${ }^{34}$. It stands to reason that the more genetically or antigenically similar two viruses are, the more likely they are to induce cross-reactive responses. For example, in mice, prior immunization to JEV leads to enhanced activation of $\mathrm{T}_{\mathrm{FH}}$ cells during DENV infection, which accelerates the development of high-avidity antibodies ${ }^{34}$. By contrast, immunity to YFV was not as efficient in inducing cross-protection, 


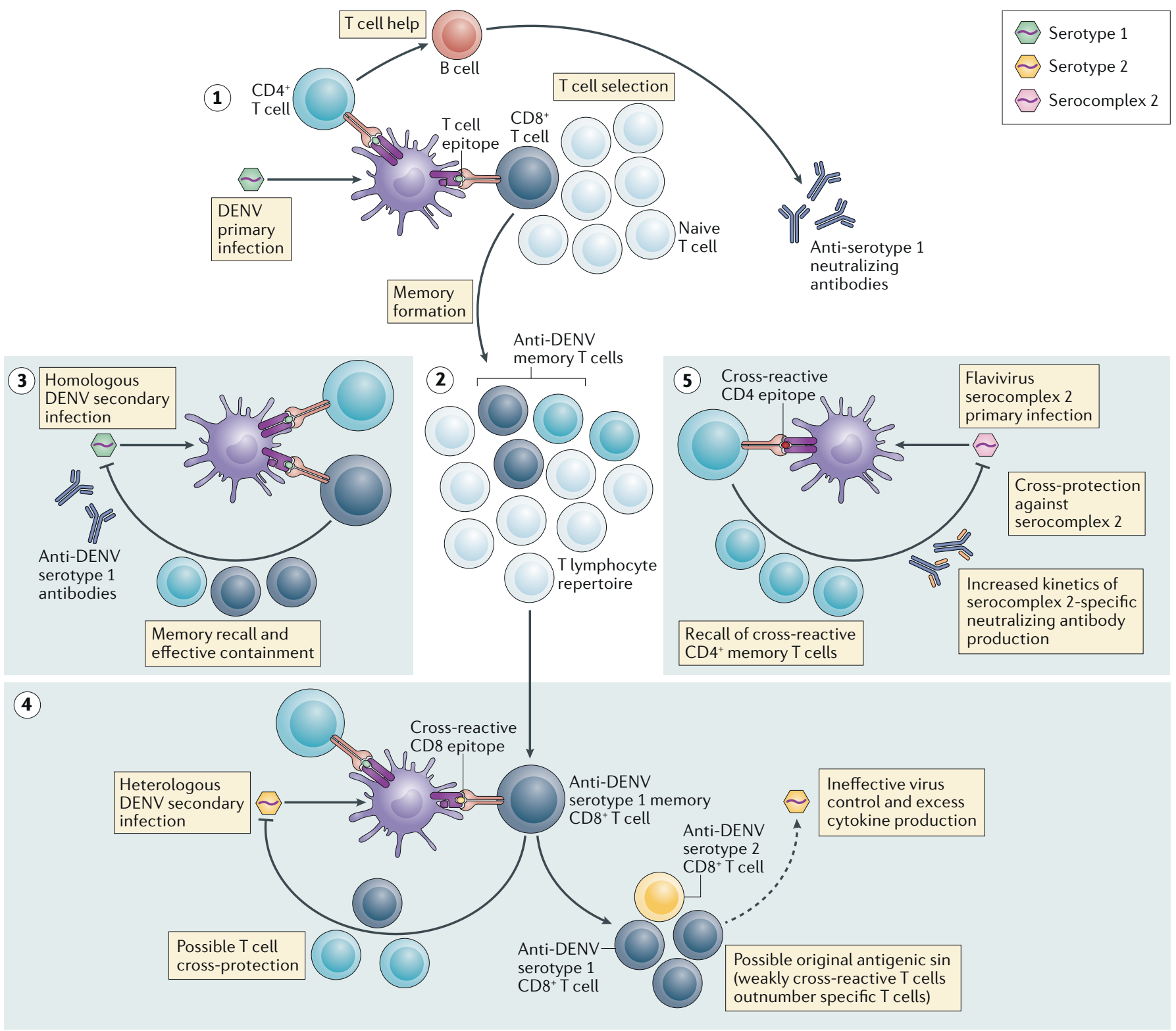

Fig. 5 | Theories of $\mathrm{T}$ cell contributions to dengue virus protection versus pathology. There is increasing evidence that T cells may contribute to both protection and pathology during dengue virus (DENV) infection. (1) A primary infection with serotype 1 of DENV leads to selection of serotype 1 -specific $\mathrm{CD} 4^{+}$and $\mathrm{CD} 8^{+} \mathrm{T}$ cells that are activated and clonally expanded. Activated $\mathrm{CD} 4^{+} \mathrm{T}$ cells provide help for $\mathrm{B}$ cells. These produce serotype 1-specific antibodies, which are efficient in clearing DENV infection. (2) Following infection resolution and memory formation, serotype 1-specific memory $\mathrm{CD}^{+}$and $\mathrm{CD} 8^{+} \mathrm{T}$ cells are retained in the T cell repertoire. (3) A secondary homologous challenge evokes a memory recall response and effective containment of infection by T cells that recognize the same MHC peptides, leading to effective infection $\operatorname{control}^{34,114}$. (4) A secondary challenge with a heterologous DENV strain, serotype 2, has the potential to reactivate cross-reactive memory T cells that are of greater specificity for serotype 1 than for serotype 2 but may also provide protection ${ }^{64,115}$. However, these serotype 1-specific memory T cells that are weakly cross-reactive may also outcompete naive T cells that would be more specific for serotype 2, resulting in original antigenic sin and the expansion of a memory T cell pool that has low specificity for serotype 2 and may contribute to poor viral clearance and excessive cytokine production ${ }^{42,113}$. Dashed arrow indicates a weak potential to control infection. (5) In the case of serocomplex cross-reactive memory, such as a primary infection with Japanese encephalitis virus followed by a secondary challenge with DENV, cross-reactive $\mathrm{CD}^{+}$memory T cells are reactivated in germinal centres, a process called memory recall, and improve both affinity and avidity of antibodies that are cross-reactive and neutralizing. These memory $T$ cells can provide cross-protection across infections with two different serocomplexes ${ }^{34}$.

potentially because it shares fewer predicted MHCbinding epitopes with DENV than $\mathrm{JEV}^{34}$. In experimental infections in primates, prior immunity to DENV was shown to increase titres of ZIKV in some studies, while others showed no significant influence ${ }^{122,123}$, potentially indicating that the timing of reinfection and/or antigenicity of the specific viruses used can influence cross-reactive responses. 
Some DENV vaccines are based on chimeric viruses, which often consist of a viral backbone that contains non-structural proteins from one virus and structural proteins from another. An example of this is Dengvaxia, a tetravalent vaccine that consists of all non-structural proteins of the YFV attenuated vaccine strain 17D, including the polymerase NS5, the capsid protein from YF-17D and two structural proteins, E and prM, from each of the four serotypes of DENV. This was designed so that the replication rates of each serotype of the vaccine strains are similar on the basis of being replicated by the same YFV polymerase ${ }^{124}$. However, this also means that immune responses against epitopes of the non-structural proteins would need to be serocomplex cross-reactive (that is, directed at multiple viruses from differing serocomplexes, such as YFV and DENV) in order to induce optimal immunity to DENV. Specifically, some cross-reactive $\mathrm{CD}^{+}$and $\mathrm{CD}^{+} \mathrm{T}$ cell epitopes in the YFV backbone may be required for anti-DENV T cell help and cytotoxicity functions. As noted above, many immunodominant protective $\mathrm{CD} 8^{+}$epitopes are found in the non-structural proteins $s^{64}$, and to exploit the activity of memory YFV-specific T cells against these epitopes, cross reactivity for DENV would be required. TAK-003 (Takeda Pharmaceutical Company), another tetravalent chimeric vaccine candidate that is currently undergoing clinical testing, is composed of a backbone derived from DENV2 and therefore does not require serocomplex cross-reactive responses to induce protective immunity to the four DENV serotypes ${ }^{125}$. The efficacy of this vaccine in humans has not yet been reported.

Besides prior immunity to flaviviruses, trained immunity to other infections can also influence how the immune system responds to flaviviruses. Trained immunity, a form of innate immune memory, results from transcriptional changes acquired by innate cell types, such as monocytes, NK cells and innate lymphoid cells upon exposure to an inflammatory insult or infection. Trained immunity can last from weeks to months and does not require classical immune memory involving adaptive immune cells ${ }^{126}$. For example, it was shown that Bacillus Calmette-Guérin (BCG) vaccination can induce epigenetic reprogramming in monocytes, and these changes influenced subsequent immune activation to YFV immunization ${ }^{127}$. These studies indicate that it is important to consider different facets of the immune status of the host in order to fully understand immune responses to flaviviruses.

First described in the context of antibody responses to influenza, this refers to the observation that during repeated infections with related viruses, immune responses are skewed towards the original strain encountered. Similar to original antigenic sin, this concept incorporates the idea that antigenic exposure to similar viruses is influenced by the genetic relationships

between them and that certain epitopes of 'senior strains' experienced early in life will begin to dominate the immune responses during subsequent exposures to related pathogens. monoclonal antibodies, it is increasingly understood that some epitopes on the virion surface have the potential to elicit cross-neutralizing antibodies to all four DENV serotypes, and these are prime antigen targets for rational vaccine design ${ }^{128}$. Recent clinical trials of Dengvaxia showed good efficacy in recipients who were seropositive for DENV but low to poor efficacy in DENV seronegative people $^{129}$. This lack of efficacy in the DENV-naive population may be due to factors such as waning titres of antibodies following vaccination or, possibly, the lack of $\mathrm{CD}^{+}$ T cell help during antibody affinity maturation upon natural DENV exposure because the vaccine backbone, which contains the majority of $\mathrm{CD} 4^{+} \mathrm{T}$ cell epitopes, is derived from YFV and is therefore mismatched. It is also possible that the epitopes in the non-structural proteins that are recognized by $\mathrm{CD}^{+}$and $\mathrm{CD} 8^{+} \mathrm{T}$ cells are not dispensable for cross-protection, which emphasizes the need for immune responses that neutralize multiple serotypes. It is difficult to design vaccines that elicit $\mathrm{T}$ cell responses because human HLAs are diverse; however, including common $\mathrm{CD} 4{ }^{+} \mathrm{T}$ cell epitopes within vaccines may promote better $\mathrm{T}_{\mathrm{FH}}$ cell responses, which may then lead to improved cross-protection against multiple DENV serotypes and to improved $\mathrm{CD}^{+} \mathrm{T}$ cell targeting of infected cells. In support of this hypothesis, $\mathrm{CD} 4^{+} \mathrm{T}$ cells were shown to contribute to vaccine-induced protection in a mouse model of DENV infection using immunocompromised mice ${ }^{49}$.

While much of the literature regarding heterotypic immunity to DENV has focused on the potential of $\mathrm{ADE}$ and original antigenic sin as mechanisms leading to increased immune pathology during acute secondary infection, there is also the possibility of immune responses to secondary infections being skewed towards epitopes that were shared by the primary and secondary challenge strains. In the context of antigenically similar families of viruses like influenza, antigenic seniority can make it difficult to direct immune responses towards epitopes that are variable between strains ${ }^{130}$. However, in the context of sequential flavivirus infections, pre-existing antibodies were shown to have improved binding (avidity) to virus during a challenge with a virus from a heterologous serocomplex, and this was dependent on the presence of crossreactive $\mathrm{T}_{\mathrm{FH}}$ cells in $\mathrm{LNs}^{34}$. Potentially, rather than being a limitation, immune-dominant epitopes could be utilized to accelerate immune recognition and cross-protection.

Given that the phenotypes of adaptive immune cells are shaped by the immune microenvironment of the skin-draining LNs close to the site of infection, not only the choice of antigen or vaccine composition but also the route of immunization ${ }^{131}$ and the polarization of DCs and $\mathrm{T}$ cells ${ }^{132}$ may be key for inducing optimal immunity. There is also growing insight into the immune mechanisms that can lead to protection from DENV or adverse outcomes such as B cell-mediated or T cell-mediated pathologies. These could, theoretically, be influenced by factors such as the antigenicity of the viruses that sequentially infected the population, the duration of time between outbreaks, virus-intrinsic factors, prior exposures to related and unrelated infectious diseases or host genetic factors, some of which are likely as-yet unidentified.

Published online 24 January 2019 
1. Bhatt, S. et al. The global distribution and burden of dengue. Nature 496, 504-507 (2013).

2. Lindenbach, B. D. \& Rice, C. M. in Fields Virology 4th edn (eds Knipe, D. M. \& Howley, P. M.) 991-104 (Lippincott Williams \& Wilkins, 2001).

3. Sabin, A. B. Research on dengue during World War II. Am. J. Trop. Med. Hyg. 1, 30-50 (1952).

4. Sangkawibha, N. et al. Risk factors in dengue shock syndrome: a prospective epidemiologic study in Rayong, Thailand. I. The 1980 outbreak. Am. J. Epidemiol. 120, 653-669 (1984).

5. Avirutnan, P., Malasit, P., Seliger, B., Bhakdi, S. \& Husmann, M. Dengue virus infection of human endothelial cells leads to chemokine production, complement activation, and apoptosis. J. Immunol. 161, 6338-6346 (1998).

6. Jessie, K., Fong, M. Y., Devi, S., Lam, S. K. \& Wong, K. T. Localization of dengue virus in naturally infected human tissues, by immunohistochemistry and in situ hybridization. J. Infect. Dis. 189, 1411-1418 (2004).

7. Aye, K. S. et al. Pathologic highlights of dengue hemorrhagic fever in 13 autopsy cases from Myanmar. Hum. Pathol. 45, 1221-1233 (2014).

8. de Andino, R. M. et al. The absence of dengue virus in the skin lesions of dengue fever. Int. J. Dermatol. 24 , 48-51 (1985).

9. Guzman, M. G., Gubler, D. J., Izquierdo, A., Martinez, E. \& Halstead, S. B. Dengue infection. Nat. Rev. Dis. Primers 2, 16055 (2016).

10. Vaughn, D. W. et al. Dengue viremia titer, antibody response pattern, and virus serotype correlate with disease severity. J. Infect. Dis. 181, 2-9 (2000).

11. Libraty, D. H. et al. High circulating levels of the dengue virus nonstructural protein NS1 early in dengue illness correlate with the development of dengue hemorrhagic fever. J. Infect. Dis. 186, 1165-1168 (2002).

12. Tricou, V., Minh, N. N., Farrar, J., Tran, H. T. \& Simmons, C. P. Kinetics of viremia and NS 1 antigenemia are shaped by immune status and virus serotype in adults with dengue. PLOS Negl. Trop. Dis. 5, e 1309 (2011).

13. Costa, V. V. et al. Subversion of early innate antiviral responses during antibody-dependent enhancement of dengue virus infection induces severe disease in immunocompetent mice. Med. Microbiol. Immunol. 203, 231-250 (2014).

14. Hotta, H. et al. Inoculation of dengue virus into nude mice. J. Gen. Virol. 52, 71-76 (1981).

15. Janssens, A. S. et al. Mast cell distribution in normal adult skin. J. Clin. Pathol. 58, 285-289 (2005)

16. Zaba, L. C., Fuentes-Duculan, J., Steinman, R. M., Krueger, J. G. \& Lowes, M. A. Normal human dermis contains distinct populations of CD $11 \mathrm{C}^{+} \mathrm{BDCA}-1^{+}$ dendritic cells and CD163+ ${ }^{+}$XIIIIA+ macrophages. J. Clin. Invest. 117, 2517-2525 (2007).

17. Libraty, D. H., Pichyangkul, S., Ajariyakhajorn, C., Endy, T. P. \& Ennis, F. A. Human dendritic cells are activated by dengue virus infection: enhancement by gamma interferon and implications for disease pathogenesis. J. Virol. 75, 3501-3508 (2001).

18. Ho, L. J. et al. Infection of human dendritic cells by dengue virus causes cell maturation and cytokine production. J. Immunol. 166, 1499-1506 (2001).

19. St John, A. L. et al. Immune surveillance by mast cells during dengue infection promotes natural killer (NK) and NKT-cell recruitment and viral clearance. Proc. Natl Acad. Sci. USA 108, 9190-9195 (2011).

20. McLachlan, J. B. et al. Mast cell-derived tumor necrosis factor induces hypertrophy of draining lymph nodes during infection. Nat. Immunol. 4 1199-1205 (2003).

21. Shelburne, C. P. et al. Mast cells augment adaptive immunity by orchestrating dendritic cell trafficking through infected tissues. Cell Host Microbe 6 331-342 (2009)

22. Taweechaisupapong, S. et al. Langerhans cell density and serological changes following intradermal immunisation of mice with dengue 2 virus. J. Med. Microbiol. 45, 138-145 (1996).

23. Randolph, G. J., Angeli, V. \& Swartz, M. A. Dendritic cell trafficking to lymph nodes through lymphatic vessels. Nat. Rev. Immunol. 5, 617-628 (2005)

24. Marchette, N. J. et al. Studies on the pathogenesis of dengue infection in monkeys. 3. Sequential distribution of virus in primary and heterologous infections. J. Infect. Dis. 128, 23-30 (1973).

25. Kyle, J. L., Beatty, P. R. \& Harris, E. Dengue virus infects macrophages and dendritic cells in a mouse model of infection. J. Infect. Dis. 195, 1808-1817 (2007).

26. Merad, M., Sathe, P., Helft, J., Miller, J. \& Mortha, A. The dendritic cell lineage: ontogeny and function of dendritic cells and their subsets in the steady state and the inflamed setting. Annu. Rev. Immunol. 31, 563-604 (2013).

27. Schmid, M. A. \& Harris, E. Monocyte recruitment to the dermis and differentiation to dendritic cells increases the targets for dengue virus replication. PLOS Pathog. 10, e1004541 (2014).

28. Cerny, D. et al. Selective susceptibility of human skin antigen presenting cells to productive dengue virus infection. PLOS Pathog. 10, e1004548 (2014). This study describes cell types and DC subsets infected by DENV in human skin explants.

29. Kwissa, M. et al. Dengue virus infection induces expansion of a $\mathrm{CD} 14^{+} \mathrm{CD} 16^{+}$monocyte population that stimulates plasmablast differentiation. Cell Host Microbe 16, 115-127 (2014).

30. St John, A. L., Rathore, A. P., Raghavan, B., Ng, M. L. ¿ Abraham, S. N. Contributions of mast cells and vasoactive products, leukotrienes and chymase, to dengue virus-induced vascular leakage. eLife $\mathbf{2}$, e00481 (2013).

31. Soundravally, R. \& Hoti, S. L. Immunopathogenesis of dengue hemorrhagic fever and shock syndrome: role of TAP and HPA gene polymorphism. Hum. Immunol. 68, 973-979 (2007)

32. LaFleur, C. et al. HLA-DR antigen frequencies in Mexican patients with dengue virus infection: HLA-DR4 as a possible genetic resistance factor for dengue hemorrhagic fever. Hum. Immunol. 63, 1039-1044 (2002).

33. Nightingale, Z. D., Patkar, C. \& Rothman, A. L. Viral replication and paracrine effects result in distinct. functional responses of dendritic cells following infection with dengue 2 virus. J. Leukoc. Biol. 84, 1028-1038 (2008)

34. Saron, W. A. A. et al. Flavivirus serocomplex cross-reactive immunity is protective by activating heterologous memory CD4 T cells. Sci. Adv. 4, eaar4297 (2018)

This study demonstrates functional $\mathrm{CD4}^{+} \mathrm{T}_{\mathrm{FH}}$ cell responses during secondary homologous dengue or following other flavivirus infections.

35. Gack, M. U. \& Diamond, M. S. Innate immune escape by dengue and West Nile viruses. Curr. Opin. Virol. 20 , 119-128 (2016)

36. Montoya, M. et al. Type I interferons produced by dendritic cells promote their phenotypic and functional activation. Blood 99, 3263-3271 (2002).

37. Wakil, A. E., Wang, Z. E., Ryan, J. C., Fowell, D. J. \& Locksley, R. M. Interferon gamma derived from CD4 ${ }^{+}$ $T$ cells is sufficient to mediate $T$ helper cell type 1 development. J. Exp. Med. 188, 1651-1656 (1998)

38. Mathew, A. et al. Dominant recognition by human CD8 ${ }^{+}$cytotoxic $T$ lymphocytes of dengue virus nonstructural proteins NS3 and NS1.2a. J. Clin. Invest. 98, 1684-1691 (1996)

39. Costa, V. V. et al. Dengue virus-infected dendritic cells, but not monocytes, activate natural killer cells through a contact-dependent mechanism involving adhesion molecules. mBio 8, e00741-17 (2017).

40. Gagnon, S. J., Ennis, F. A. \& Rothman, A. L. Bystander target cell lysis and cytokine production by dengue virus-specific human $\mathrm{CD}^{+}$cytotoxic T-lymphocyte clones. J. Virol. 73, 3623-3629 (1999).

41. Matangkasombut, P. et al. Invariant NKT cell response to dengue virus infection in human. PLOS Negl. Trop. Dis. 8, e2955 (2014).

42. Mongkolsapaya, J. et al. Original antigenic sin and apoptosis in the pathogenesis of dengue hemorrhagic fever. Nat. Med. 9, 921-927 (2003). This paper describes a contribution of $\mathrm{T}$ cell original antigenic sin to severe dengue disease.

43. Screaton, G., Mongkolsapaya, J., Yacoub, S. \& Roberts, C. New insights into the immunopathology and control of dengue virus infection. Nat. Rev. Immunol. 15, 745-759 (2015).

44. Hatch, S. et al. Intracellular cytokine production by dengue virus-specific T cells correlates with subclinical secondary infection. J. Infect. Dis. 203, 1282-1291 (2011).

45. Gwinn, W., Sun, W., Innis, B. L., Caudill, J. \& King, A. D. Serotype-specific $T(H) 1$ responses in recipients of two doses of candidate live-attenuated dengue virus vaccines. Am. J. Trop. Med. Hyg. 69, 39-47 (2003).

46. Rey, F. A., Stiasny, K., Vaney, M. C., Dellarole, M. \& Heinz, F. X. The bright and the dark side of human antibody responses to flaviviruses: lessons for vaccine design. EMBO Rep. 19, 206-224 (2018).

47. Simon-Loriere, E. et al. Increased adaptive immune responses and proper feedback regulation protect against clinical dengue. Sci. Trans/ Med. 9, eaal5088 (2017). This study highlights that transcriptional signatures of $\mathrm{CD4}^{+} \mathrm{T}$ cell activation and enhanced antigen presentation are present during human asymptomatic dengue infection, suggesting adaptive immune control of dengue

48. Weiskopf, D. et al. HLA-DRB1 alleles are associated with different magnitudes of dengue virus-specific CD4 T-cell responses. J. Infect. Dis. 214, 1117-1124 (2016)

49. Yauch, L. E. et al. CD4 ${ }^{+} T$ cells are not required for the induction of dengue virus-specific $\mathrm{CD}^{+} \mathrm{T}$ cell or antibody responses but contribute to protection after vaccination. J. Immunol. 185, 5405-5416 (2010).

50. Longhi, M. P. et al. Dendritic cells require a systemic type I interferon response to mature and induce CD4 Th1 immunity with poly IC as adjuvant. J. Exp. Med. 206, 1589-1602 (2009)

51. Le Bon, A. et al. Cross-priming of CD8+ T cells stimulated by virus-induced type I interferon. Nat. Immunol. 4, 1009-1015 (2003)

52. Luhn, K. et al. Increased frequencies of CD4+ CD25(high) regulatory T cells in acute dengue infection. J. Exp. Med. 204, 979-985 (2007).

53. Jayaratne, H. E. et al. Regulatory T cells in acute dengue viral infection. Immunology 154, 89-97 (2018).

54. Haltaufderhyde, K. et al. Activation of peripheral T follicular helper cells during acute dengue virus infection. J. Infect. Dis. 218, 1675-1685 (2018).

55. Shulman, Z. et al. T follicular helper cell dynamics in germinal centers. Science 341, 673-677 (2013).

56. Kurane, I., Meager, A. \& Ennis, F. A. Dengue virus-specific human $\mathrm{T}$ cell clones. Serotype crossreactive proliferation, interferon gamma production, and cytotoxic activity. J. Exp. Med. 170, 763-775 (1989).

57. Weiskopf, D. et al. Dengue virus infection elicits highly polarized $\mathrm{CX} 3 \mathrm{CR} 1{ }^{+}$cytotoxic $\mathrm{CD} 4^{+} \mathrm{T}$ cells associated with protective immunity. Proc. Natl Acad. Sci. USA 112, E4256-E4263 (2015)

58. Tian, Y. et al. Unique phenotypes and clonal expansions of human CD4 effector memory T cells re-expressing CD45RA. Nat. Commun. 8, 1473 (2017). References 57 and 58 suggest a protective role for effector memory $\mathrm{CD4}^{+} \mathrm{T}$ cells known as $\mathrm{T}_{\mathrm{EMRA}}$ cells, which are cytotoxic in nature, during secondary dengue

59. Friberg, $\mathrm{H}$. et al. Cross-reactivity and expansion of dengue-specific $\mathrm{T}$ cells during acute primary and secondary infections in humans. Sci. Rep. 1, 51 (2011).

60. Chandele, A. et al. Characterization of human CD8 $\mathrm{T}$ cell responses in dengue virus-infected patients from India. J. Virol. 90, 11259-11278 (2016).

61. Singla, M. et al. Immune response to dengue virus infection in pediatric patients in New Delhi, Indiaassociation of viremia, inflammatory mediators and monocytes with disease severity. PLOS Negl. Trop. Dis. 10, e0004497 (2016).

62. Rivino, L. et al. Virus-specific T lymphocytes home to the skin during natural dengue infection. Sci. Transl Med. 7, 278ra235 (2015).

63. Hughes, A. L. Evolutionary change of predicted cytotoxic T cell epitopes of dengue virus. Infect. Genet Evol. 1, 123-130 (2001).

64. Weiskopf, D. et al. Comprehensive analysis of dengue virus-specific responses supports an HLA-linked protective role for $\mathrm{CD}^{+} \mathrm{T}$ cells. Proc. Natl Acad. Sci. USA 110, E2046-E2053 (2013). This study suggests a protective role for $\mathrm{CDB}^{+}$ T cells during human dengue disease.

65. Rivino, L. et al. Differential targeting of viral components by $\mathrm{CD} 4^{+}$versus $\mathrm{CD} 8^{+} \mathrm{T}$ lymphocytes in dengue virus infection. J. Virol. 87, 2693-2706 (2013).

66. Grifoni, A. et al. Global assessment of dengue virusspecific $\mathrm{CD}^{+} \mathrm{T}$ cell responses in dengue-endemic areas. Front. Immunol. 8, 1309 (2017). This multi-cohort study reports immunodominant epitopes of dengue-specific human $\mathrm{CD4}^{+} \mathrm{T}$ cells.

67. Balakrishnan, T. et al. Dengue virus activates polyreactive, natural IgC B cells after primary and secondary infection. PLOS ONE 6, e29430 (2011).

68. De Milito, A. et al. Mechanisms of hypergammaglobulinemia and impaired antigen-specific humoral immunity in HIV-1 infection. Blood 103, 2180-2186 (2004).

69. Mathew, A. et al. B cell responses during primary and secondary dengue virus infections in humans. J. Infect. Dis. 204, 1514-1522 (2011).

70. Mehlhop, E. et al. Complement protein C1 q reduces the stoichiometric threshold for antibody-mediated neutralization of West Nile virus. Cell Host Microbe 6 , 381-391 (2009)

71. Dowd, K. A. \& Pierson, T. C. Antibody-mediated neutralization of flaviviruses: a reductionist view. Virology 411, 306-315 (2011).

72. Rouvinski, A. et al. Recognition determinants of broadly neutralizing human antibodies against dengue viruses. Nature 520, 109-113 (2015). 
73. Fibriansah, G. et al. Cryo-EM structure of an antibody that neutralizes dengue virus type 2 by locking $\mathrm{E}$ protein dimers. Science 349, 88-91 (2015). References 72 and 73 identify broadly neutralizing dengue antibodies targeting the $\mathrm{E}$ dimer of DENV.

74. Cockburn, J. J. et al. Mechanism of dengue virus broad cross-neutralization by a monoclonal antibody. Structure 20, 303-314 (2012)

75. Lok, S. M. The interplay of dengue virus morphological diversity and human antibodies. Trends Microbiol. 24 284-293 (2016)

76. Waggoner, J. J. et al. Homotypic dengue virus reinfections in Nicaraguan children. J. Infect. Dis. 214 986-993 (2016)

77. Friberg, $\mathrm{H}$. et al. Memory $\mathrm{CD} 8^{+} \mathrm{T}$ cells from naturally acquired primary dengue virus infection are highly cross-reactive. Immunol. Cell Biol. 89, 122-129 (2011).

78. Mangada, M. M. \& Rothman, A. L. Altered cytokine responses of dengue-specific $C D 4^{+} \mathrm{T}$ cells to heterologous serotypes. J. Immunol. 175, 2676-2683 (2005).

79. Beaumier, C. M., Mathew, A., Bashyam, H. S. \& Rothman, A. L Cross-reactive memory $\mathrm{CD} 8^{+} \mathrm{T}$ cells alter the immune response to heterologous secondary dengue virus infections in mice in a sequence-specific manner. J. Infect. Dis. 197, 608-617 (2008).

80. Wrammert, J. et al. Rapid cloning of high-affinity human monoclonal antibodies against influenza virus. Nature 453, 667-671 (2008)

81. Wrammert, J. et al. Rapid and massive virus-specific plasmablast responses during acute dengue virus infection in humans. J. Virol. 86, 2911-2918 (2012).

82. Priyamvada, L. et al. B cell responses during secondary dengue virus infection are dominated by highly cross-reactive, memory-derived plasmablasts. J. Virol. 90, 5574-5585 (2016).

83. $\mathrm{Xu}, \mathrm{M}$. et al. Plasmablasts generated during repeated dengue infection are virus glycoprotein-specific and bind to multiple virus serotypes. J. Immunol. 189 5877-5885 (2012)

84. Townsley, E. et al. Distinct activation phenotype of a highly conserved novel HLA-B57-restricted epitope during dengue virus infection. Immunology 141 27-38 (2014)

85. Guzman, M. G. et al. Epidemiologic studies on dengue in Santiago de Cuba, 1997. Am. J. Epidemiol. 152, 793-799; discussion 804 (2000).

86. Chau, T. N. et al. Dengue in Vietnamese infantsresults of infection-enhancement assays correlate with age-related disease epidemiology, and cellular immune responses correlate with disease severity. J. Infect. Dis. 198, 516-524 (2008).

87. Katzelnick, L. C. et al. Antibody-dependent enhancement of severe dengue disease in humans. Science 358, 929-932 (2017). This paper supports the concept of ADE of dengue disease in humans.

88. Halstead, S. B. \& O'Rourke, E. J. Dengue viruses and mononuclear phagocytes. I. Infection enhancement by non-neutralizing antibody. J. Exp. Med. 146 201-217 (1977)

This paper describes the mechanism of ADE of dengue infection, involving uptake of virus particles, dependent on the Fc region of antibodies.

89. de Alwis, R. et al. Dengue viruses are enhanced by distinct populations of serotype cross-reactive antibodies in human immune sera. PLOS Pathog. 10 , e1004386 (2014).

90. Zellweger, R. M., Prestwood, T. R. \& Shresta, S Enhanced infection of liver sinusoidal endothelial cells in a mouse model of antibody-induced severe dengue disease. Cell Host Microbe 7, 128-139 (2010).

91. Goncalvez, A. P., Engle, R. E., St Claire, M., Purcell, R. H. \& Lai, C. J. Monoclonal antibody-mediated enhancement of dengue virus infection in vitro and in vivo and strategies for prevention. Proc. Natl Acad. Sci. USA 104, 9422-9427 (2007)

92. Syenina, A., Jagaraj, C. J., Aman, S. A., Sridharan, A. $\&$ St John, A. L. Dengue vascular leakage is augmented by mast cell degranulation mediated by immunoglobulin Fcgamma receptors. eLife 4, e05291 (2015). This study provides evidence that antibodydependent mast cell activation can contribute to vascular leakage during DENV infection.

93. Rodenhuis-Zybert, I. A. et al. A fusion-loop antibody enhances the infectious properties of immature flavivirus particles. J. Virol. 85, 11800-11808 (2011).

94. Dejnirattisai, W. et al. Cross-reacting antibodies enhance dengue virus infection in humans. Science $\mathbf{3 2 8}$, 745-748 (2010)

95. Pierson, T. C. et al. The stoichiometry of antibodymediated neutralization and enhancement of West Nile virus infection. Cell Host Microbe 1, 135-145 (2007).
This study reports the number of antibodies required to neutralize or exacerbate flavivirus infection.

96. Tsai, T. T. et al. Antibody-dependent enhancement infection facilitates dengue virus-regulated signaling of IL-10 production in monocytes. PLOS Negl. Trop. Dis. 8, e3320 (2014)

97. Kou, Z. et al. Human antibodies against dengue enhance dengue viral infectivity without suppressing type I interferon secretion in primary human monocytes. Virology 410, 240-247 (2011).

98. Garcia-Bates, T. M. et al. Association between magnitude of the virus-specific plasmablast response and disease severity in dengue patients. J. Immunol. 190, 80-87 (2013)

99. Joo, H. et al. Serum from patients with SLE instructs monocytes to promote IgG and IgA plasmablast differentiation. J. Exp. Med. 209, 1335-1348 (2012).

100. Wang, T. T. et al. IgG antibodies to dengue enhanced for FcgammaRIIIA binding determine disease severity. Science 355, 395-398 (2017).

This study shows that dengue-specific afucosylated IgG1 antibodies are associated with severe dengue.

101. Israel, E. J et al. Expression of the neonatal Fc receptor FcRn, on human intestinal epithelial cells. Immunology 92, 69-74 (1997).

102. Chau, T. N. et al. Dengue virus infections and maternal antibody decay in a prospective birth cohort study of Vietnamese infants. J. Infect. Dis. 200, 1893-1900 (2009).

This paper discusses maternal antibody decay and the associated risk of severe dengue disease in infants.

103. Clapham, H. et al. Epidemiology of infant dengue cases illuminates serotype-specificity in the interaction between immunity and disease, and changes in transmission dynamics. PLOS Negl. Trop. Dis. 9 e0004262 (2015)

104. Libraty, D. H. et al. A prospective nested case-control study of dengue in infants: rethinking and refining the antibody-dependent enhancement dengue hemorrhagic fever model. PLOS Med. 6, e1000171 (2009).

105. Ng, J. K. et al. First experimental in vivo model of enhanced dengue disease severity through maternally acquired heterotypic dengue antibodies. PLOS Pathog 10, e1004031 (2014)

106. Lee, P. X., Ong, L. C., Libau, E. A. \& Alonso, S. Relative contribution of dengue IgG antibodies acquired during gestation or breastfeeding in mediating dengue disease enhancement and protection in type I interferon receptor-deficient mice. PLOS Negl. Trop. Dis. 10, e0004805 (2016).

107. Balsitis, S. J. et al. Lethal antibody enhancement of dengue disease in mice is prevented by Fc modification. PLOS Pathog. 6, e1000790 (2010).

108. Chan, K. R. et al. Cross-reactive antibodies enhance live attenuated virus infection for increased immunogenicity. Nat. Microbiol. 1, 16164 (2016).

109. Sridhar, S. et al. Effect of dengue serostatus on dengue vaccine safety and efficacy. N. Engl. J. Med. 379, 327-340 (2018).

This study compiles data from multiple Dengvaxia clinical trials that showed efficacy against dengue and reports a risk of hospitalization and severe dengue for vaccinated DENV-naive individuals.

110. Halstead, S. B. Dengvaxia sensitizes seronegatives to vaccine enhanced disease regardless of age. Vaccine 35, 6355-6358 (2017).

111. Koraka, P. et al. Elevated levels of total and dengue virus-specific immunoglobulin $\mathrm{E}$ in patients with varying disease severity. J. Med. Virol. 70, 91-98 (2003).

112. Kurane, I., Hebblewaite, D., Brandt, W. E. \& Ennis, F. A Lysis of dengue virus-infected cells by natural cellmediated cytotoxicity and antibody-dependent cellmediated cytotoxicity. J. Virol. 52, 223-230 (1984).

113. Culshaw, A. et al. Germline bias dictates cross-serotype reactivity in a common dengue-virus-specific $\mathrm{CD} 8^{+} \mathrm{T}$ cell response. Nat. Immunol. 18, 1228-1237 (2017). This study reports that bias towards certain TCRMHC-peptide interactions can lead to increased DENV pathology during secondary infections, indicating possible germline-encoded susceptibility to severe disease.

114. Yauch, L. E. et al. A protective role for dengue virusspecific CD8 ${ }^{+} \mathrm{T}$ cells. J. Immunol. 182, 4865-4873 (2009).

115. Zompi, S., Santich, B. H., Beatty, P. R. \& Harris, E. Protection from secondary dengue virus infection in a mouse model reveals the role of serotype crossreactive B and T cells. J. Immunol. 188, 404-416 (2012).

116. Yachi, P. P., Ampudia, J., Zal, T. \& Gascoigne, N. R. Altered peptide ligands induce delayed CD8-T cell receptor interaction-a role for CD8 in distinguishing antigen quality. Immunity 25, 203-211 (2006).

117. Imrie, A. et al. Differential functional avidity of dengue virus-specific $T$ cell clones for variant peptides representing heterologous and previously encountered serotypes. J. Virol. 81, 10081-10091 (2007).

118. Dung, N. T. et al. Timing of CD8 ${ }^{+} \mathrm{T}$ cell responses in relation to commencement of capillary leakage in children with dengue. J. Immunol. 184, 7281-7287 (2010).

119. Shedlock, D. J. \& Shen, H. Requirement for CD4 T cell help in generating functional CD8 T cell memory. Science 300, 337-339 (2003).

120. Kuno, G., Chang, G. J., Tsuchiya, K. R., Karabatsos, N. $\Sigma$ Cropp, C. B. Phylogeny of the genus flavivirus. J. Virol. 72, 73-83 (1998).

121. Calisher, C. H. et al. Antigenic relationships between flaviviruses as determined by cross-neutralization tests with polyclonal antisera. J. Gen. Virol. 70, 37-43 (1989).

122. George, J. et al. Prior exposure to zika virus significantly enhances peak dengue- 2 viremia in rhesus macaques. Sci. Rep. 7, 10498 (2017).

123. McCracken, M. K. et al. Impact of prior flavivirus immunity on Zika virus infection in rhesus macaques. PLOS Pathog. 13, e1006487 (2017).

124. Guy, B. et al. Preclinical and clinical development of YFV 17D-based chimeric vaccines against dengue, West Nile and Japanese encephalitis viruses. Vaccine 28, 632-649 (2010).

125. Saez-Llorens, X. et al. Immunogenicity and safety of one versus two doses of tetravalent dengue vaccine in healthy children aged 2-17 years in Asia and Latin America: 18-month interim data from a phase 2 randomised, placebo-controlled study. Lancet Infect. Dis. 18, 162-170 (2018)

126. Netea, M. G. et al. Trained immunity: a program of innate immune memory in health and disease. Science 352, aaf1098 (2016).

127. Arts, R. J. W. et al. BCG vaccination protects against experimental viral infection in humans through the induction of cytokines associated with trained immunity. Cell Host Microbe 23, 89-100 (2018).

128. Rouvinski, A et al. Covalently linked dengue virus envelope glycoprotein dimers reduce exposure of the immunodominant fusion loop epitope. Nat. Commun. 8, 15411 (2017)

129. Arredondo-Garcia, J. L. et al. Four-year safety follow-up of the tetravalent dengue vaccine efficacy randomized controlled trials in Asia and Latin America. Clin. Microbiol. Infect. 24, 755-763 (2018)

130. Lessler, J. et al. Evidence for antigenic seniority in influenza A (H3N2) antibody responses in southern China. PLOS Pathog. 8, e1002802 (2012).

131. Koutsonanos, D. G. et al. Enhanced immune responses by skin vaccination with influenza subunit vaccine in young hosts. Vaccine 33, 4675-4682 (2015)

132. Querec, T. et al. Yellow fever vaccine YF-17D activates multiple dendritic cell subsets via TLR2, 7, 8, and 9 to stimulate polyvalent immunity. J. Exp. Med. 203 413-424 (2006)

133. Vaughn, D. W. et al. Dengue in the early febrile phase: viremia and antibody responses. J. Infect. Dis. 176, 322-330 (1997).

134. St John, A. L., Abraham, S. N. \& Gubler, D. J. Barriers to preclinical investigations of anti-dengue immunity and dengue pathogenesis. Nat. Rev. Microbiol. 11, 420-426 (2013)

135. Halstead, S. B. Dengue. Lancet 370, 1644-1652 (2007).

136. Rothman, A. L. Immunity to dengue virus: a tale of original antigenic sin and tropical cytokine storms. Nat. Rev. Immunol. 11, 532-543 (2011).

\section{Acknowledgements}

The authors acknowledge funding from the National Medical Research Council of Singapore (NMRC/CBRG/0084/2015), the National Research Foundation of Singapore (NRF2016NRFCRP001-063) and Duke-National University of Singapore (NUS) Medical School to A.L.S.

Author contributions

The authors contributed equally to all aspects of the article.

\section{Competing interests}

The authors declare no competing interests.

\section{Publisher's note}

Springer Nature remains neutral with regard to jurisdictional claims in published maps and institutional affiliations.

\section{Reviewer information}

Nature Reviews Immunology thanks S. Halstead, A. Sette, and other anonymous reviewer(s) for their contribution to the peer review of this work. 